\title{
Computing Optical Properties of Ultra-thin Crystals
}

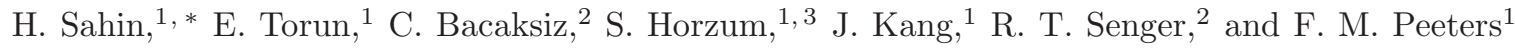 \\ ${ }^{1}$ Department of Physics, University of Antwerp, Groenenborgerlaan 171, 2020 Antwerp, Belgium \\ ${ }^{2}$ Department of Physics, Izmir Institute of Technology, 35430 Izmir, Turkey \\ ${ }^{3}$ Department of Engineering Physics, Faculty of Engineering, Ankara University, 06100 Ankara, Turkey
}

(Dated: September 16, 2021)

\begin{abstract}
An overview is given of recent advances in experimental and theoretical understanding of optical properties of ultra-thin crystal structures (graphene, phosphorene, silicene, $\mathrm{MoS}_{2}, \mathrm{MoSe}_{2}, \mathrm{WS}_{2}$, $\mathrm{WSe}_{2}$, h-AlN, h-BN, fluorographene, graphane). Ultra-thin crystals are atomically-thick layered crystals that have unique properties which differ from their 3D counterpart. Because of the difficulties in the synthesis of few-atom-thick crystal structures, which are thought to be the main building blocks of future nanotechnology, reliable theoretical predictions of their electronic, vibrational and optical properties are of great importance. Recent studies revealed the reliable predictive power of existing theoretical approaches based on density functional theory (DFT).

PACS numbers: 73.20.Hb, 82.45.Mp, 73.61.-r, 73.90.+f, 74.78.Fk
\end{abstract}

\section{INTRODUCTION}

Over the past decade, advances in materials science have shown that materials can have remarkably different properties when its dimensions are reduced. In particular, the successful isolation of graphene in 2004 [1] uncovered a new class of materials; "two dimensional (2D) atomically-thin crystal structures". 2] Following the rapid development of both top-down and bottom-up synthesis approaches in recent years, more and more members have joined the family of ultra-thin $2 \mathrm{D}$ crystals, such as graphene derivatives (graphane, grapheneoxide, fluorographene and chlorographene), hexagonal BN and AlN, silicene, transition-metal chalcogenides (with formula $\mathrm{MX}_{2}$ where $\mathrm{M}=\mathrm{Mo}, \mathrm{W}, \mathrm{Re}$ and $\mathrm{X}=\mathrm{S}, \mathrm{Se}, \mathrm{Te}$ ), black phosphorus and metal hydroxides $\left(\mathrm{Mg}(\mathrm{OH})_{2}\right.$ and $\left.\mathrm{Ca}(\mathrm{OH})_{2}\right)$. Many groups reported that in addition to their structural stability, 2D crystals also show a variety of exceptional electronic and optical properties. Despite the large amount of work that has been done on the structural and electronic properties of 2D materials, theoretical and experimental research on their optical properties are in their early stage. In this overview, following a brief introduction to experimental efforts on 2D crystals, we focus on computational methods and the most recent theoretical findings of their optical properties.

Experimentally, one of the first successful determinations of the optical reflectivity and transmission of graphene was performed by Nair et al. [3] As shown in Figures 1(a) and (b), the reflection of single layer graphene is very low, less than $0.1 \%$ in the visible region. However, its optical absorption $A=\pi \alpha=2.3 \%$ is considerable, which is practically constant over the visible spectrum, where $\alpha$ is the fine structure constant $1 / 137$. In addition, in multi-layer graphene, the optical absorption is proportional to the number of layers, i.e., each graphene

\footnotetext{
* hasan.sahin@uantwerpen.be
}

layer contributes $2.3 \%$ and the overall absorption is the superposition of those monolayers. However, such simple result may break down in the non-visible regions. In the work of Mak et al. 4. two important aspects in the optical spectrum of graphene are: (i) possible many-body effects leading to a reduction of the optical conductivity at lower photon energies, and (ii) deviations from the linear dispersion that leads to an increase of the optical absorption at high energies.

Not only the absorption properties but also other topics related to its optical properties of graphene are under intensive investigations. Sun et al. [5] reported the first ultrafast spectroscopy of hot Dirac quasiparticles in epitaxial graphene in the region near the Fermi level. In the same year, infrared spectroscopy measurements performed by Wang et al. [6] revealed that monolayers and bilayers of graphene have strong interband transitions and that their optical transitions can be substantially modified through electrical gating, much like electrical transport in field-effect transistors. This gate dependence of the interband transitions is an interesting tuning parameter for optically probing of the band structure of graphene. For monolayer graphene, it yields directly the linear band dispersion of Dirac fermions, whereas in bilayer graphene, a dominating van Hove singularity arising from interlayer coupling is observed. The strong and layer-dependent optical transitions of graphene and the tunability by simple electrical gating holds promise for new applications in infrared optics and optoelectronics. In the work of Xia et al. [7] ultrafast photocurrent response measurements using a $1.55 \mu \mathrm{m}$ excitation laser revealed a light intensity modulation with frequencies up to $40 \mathrm{GHz}$ in single and few-layers graphene field-effecttransistors (FETs). They also concluded that the intrinsic bandwidth of such a graphene FET may lead to graphene based photodetectors that exceed $500 \mathrm{GHz}$.

Although graphene is a semi-metal in the monolayer form, it can exhibit photoluminescence (PL) by inducing a bandgap. Basically there are two approaches to open a bandgap in graphene. One is cutting graphene 

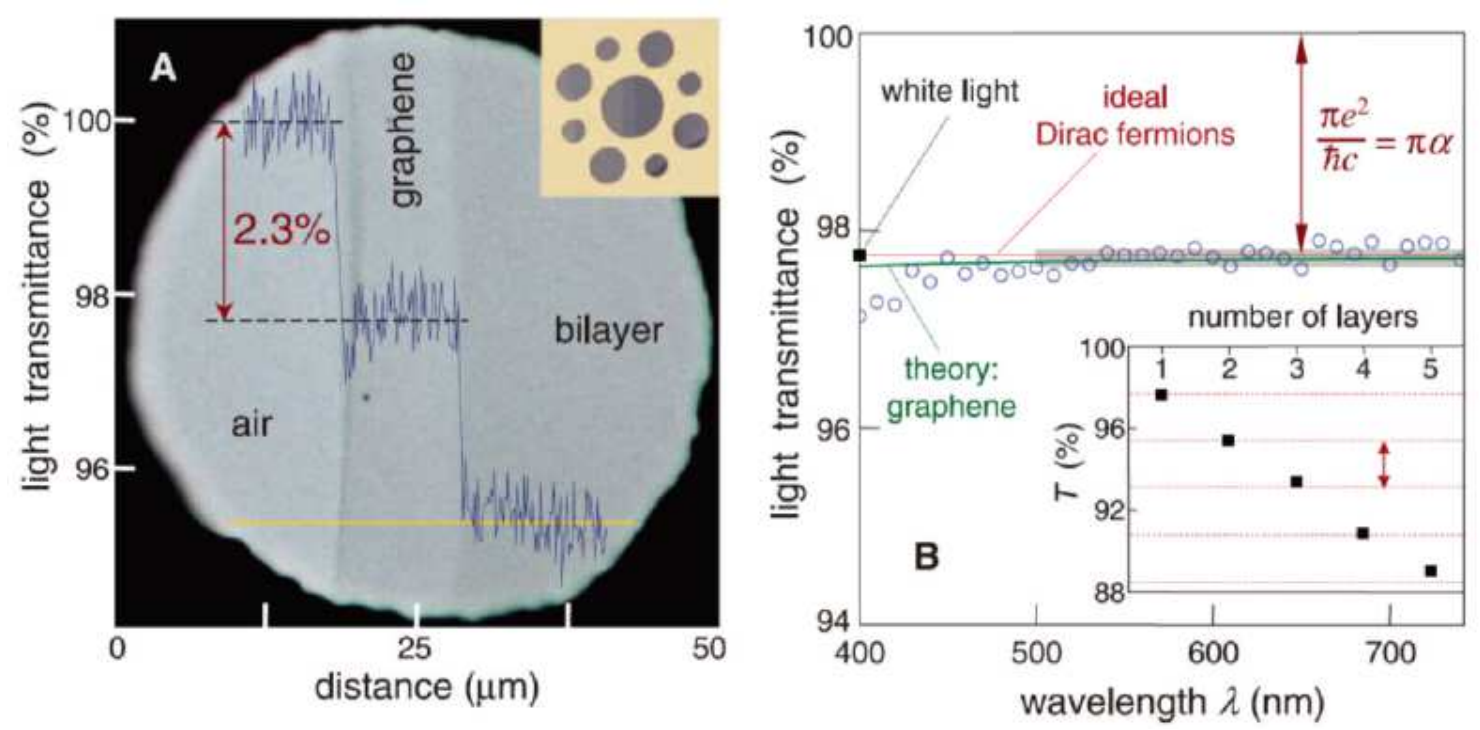

FIG. 1. (a) Photograph of a 50- $\mu m$ aperture partially covered by graphene and its bilayer. (b) Transmittance spectrum of single-layer graphene (open circles) and theoretically predicted results for two-dimensional Dirac fermions. Inset shows the transmittance of white light as a function of the number of graphene layers (squares) [from Ref. 3].

into nanoribbons and quantum dots, so that quantum confinement results in a gap opening. Pan et al. prepared water-soluble graphene quantum dots that exhibit bright blue PL by hydrothermal (chemical) cutting of oxidized graphene sheets. [8] Jin et al. further showed that the PL emission of graphene dots can be tuned through the charge transfer effect of functional groups. [9] The other way to open a gap in graphene is by chemical or physical treatments which can reduce the connectivity of the $\pi$-electron network. For instance, by using an oxygen plasma treatment, Gokus et al. observed strong PL in single-layer graphene.[10] Moreover, Eda et al. reported blue PL from chemically derived grapheneoxide. [11] Chien et al. demonstrated that it was possible to tune the PL in grapheneoxide suspensions from red to blue emission through the reduction of the surface oxide groups 12], which could thus gradually change the ratio between $\mathrm{sp}^{2}$ - and $\mathrm{sp}^{3}$-bonded carbon atoms .

The optical properties of other ultra-thin crystals than graphene has also attracted a lot of attention. The inplane dielectric function of four different TMCs: $\mathrm{MoS}_{2}$, $\mathrm{MoSe}_{2}, \mathrm{WS}_{2}$ and $\mathrm{WSe}_{2}$ were determined by Li et al. [13. through reflectance measurements at room temperature. They concluded that for all four TMC monolayers, the two lowest energy peaks in the reflectance spectrum correspond to excitonic features that stem from interband transitions at the $\mathrm{K}\left(\mathrm{K}^{\prime}\right)$ point, while at higher photon energies the spectral broad response originates from higher-lying interband transitions. Island et al. [14] reported an ultrahigh photoresponse up to $2910 \mathrm{~A} \mathrm{~W}^{-1}$ and fast switching times of about $4 \mathrm{~ms}$ with a cutoff frequency of $1000 \mathrm{~Hz}$ in a $\mathrm{TiS}_{3}$ field effect transistor (see Figure 21). $\mathrm{TiS}_{3}$ is also proven to be suitable for hydro- gen photogeneration under visible light. 15]

For TMC semiconductors, measurement of PL is one of the most efficient ways to find out their characteristics. In early experimental studies on TMCs, Mak et al. 16] and Splendiani et al. 17] showed that bulk $\mathrm{MoS}_{2}$ which exhibits PL, displays a strong peak intensity increase when the crystal is thinned down to a monolayer. The increasing PL intensity was explained as a consequence of an indirect to direct bandgap transition when going from multi to monolayer graphene. Subsequently similar indirect-to-direct bandgap crossover has been reported for other TMCs such as $\mathrm{MoSe}_{2}$ [18, 19], WSe 2 [18] and $\mathrm{WS}_{2}[20$.

In conventional semiconductors, increasing temperature or introducing defects usually reduces the PL strength. Interestingly, studies by Tongay et al. showed the opposite behavior in TMCs. 19, 21] They found that the PL of few-layer $\mathrm{MoSe}_{2}$ was much enhanced with increasing temperature, due to the thermally driven crossover from indirect towards a direct bandgap. [19] In another work, they observed an increase in the overall PL intensity as a result of the generation of vacancies in monolayer TMCs when put in a gas environment (such as $\mathrm{N}_{2}$ ). 21] The interaction between the gas molecules and the defect sites, which caused a transition of exciton population from charged to neutral excitons, was responsible for the PL enhancement. In monolayer TMCs, due to the reduced screening of the Coulomb interaction, the exciton binding energy is much larger than in bulk materials. Ugeda et al. reported an exciton binding energy of 0.55 $\mathrm{eV}$ for monolayer $\mathrm{MoSe}_{2}$ by means of scanning tunneling spectroscopy and the two-particle exciton transition energy using PL spectroscopy. 22.

In addition, many honeycomb lattice TMCs such as 

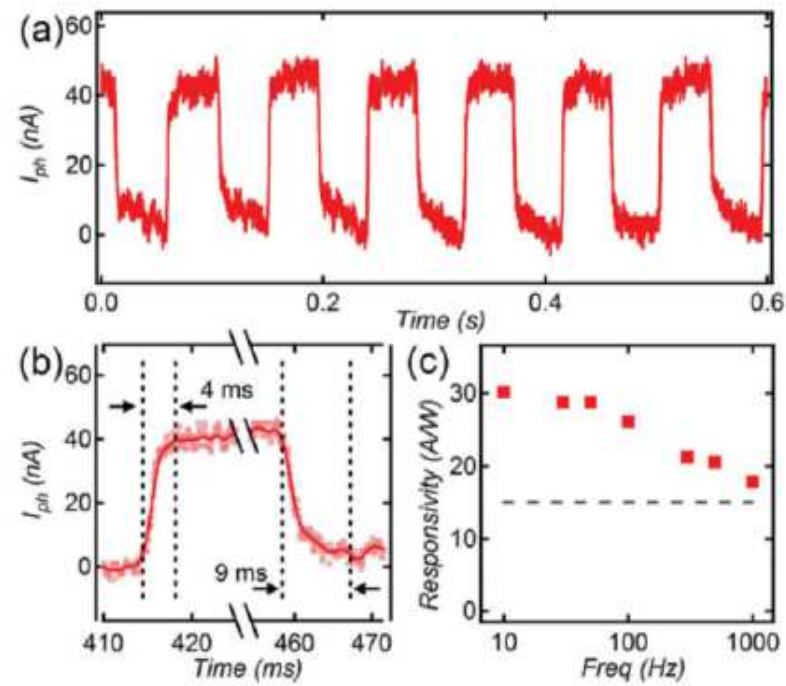

FIG. 2. (a) Current response of $\mathrm{TiS}_{3}$ field effect transistor under a $10 \mathrm{~Hz}$ mechanically modulated optical excitation. (b) Zoom on a single switching cycle at $10 \mathrm{~Hz}$ frequency. (c) Responsivity versus increasing modulation frequency for an excitation wavelength of $640 \mathrm{~nm}$ at $500 \mu \mathrm{W}$ [from Ref. 14].

$\mathrm{MoS}_{2}$ and $\mathrm{WSe}_{2}$ showed rich valley-polarized optical properties. The inequivalent valleys in the Brillouin zone could be selectively addressed using circularly-polarized light fields. 23 25] Jones et al. 25] reported the experimental generation and detection of valley coherence in $\mathrm{WSe}_{2}$. Their study was based on the fact that excitons localized in a single valley emit circularly polarized photons. Linear polarization can only be generated through recombination of an exciton which is in a coherent superposition of the two valley states. Furthermore, Srivastava et al. realized a valley Zeeman effect in $\mathrm{WSe}_{2} \cdot[26]$ They showed strong anisotropic lifting of the degeneracy of the valley pseudospin degree of freedom by using an external magnetic field.

Most recent experimental efforts have also revealed that synthesis of heterostructures made of monolayer crystal structures can be achieved such as van der Waals heterostructures made of TMC monolayers which exhibit novel optical properties. Tongay et al. reported the realization of large-area (tens of micrometers) heterostructures of CVD-grown $\mathrm{WS}_{2}$ and $\mathrm{MoS}_{2}$ monolayers. 27] They showed that the interlayer coupling can be tuned by vacuum thermal annealing. As the coupling strength increases, the photoluminescence gradually changes from a superposition of the spectra of $\mathrm{MoS}_{2}$ and $\mathrm{WS}_{2}$ monolayers to the coupled spectrum, in which charged exciton (also called trions) recombination and phonon-assisted indirect-bandgap transition dominate the light emission. Gong et al. also observed interlayer exciton transition in a $\mathrm{MoS}_{2} / \mathrm{WS}_{2}$ heterostructure, which leads to an additional PL peak at $1.4 \mathrm{eV}$ which is shown in Figure 3, 28] Similar interlayer exciton transition in $\mathrm{MoSe}_{2} / \mathrm{WSe}_{2}$ heterostructure was also reported by Rivera et al., and the interlayer exciton had a long lifetime of $1.8 \mathrm{~ns}$, an order of magnitude longer than intralayer excitons. 29] Besides their PL properties, Huo et al. demonstrated enhanced optoelectronic performances of multilayer $\mathrm{MoS}_{2} / \mathrm{WS}_{2}$ heterostructure transistors, whose photoresponsivity is three orders of magnitude larger than for devices made of monolayer $\mathrm{MoS}_{2} \cdot[30]$ Several studies have reported the observation of a photovoltaic response in $\mathrm{MoS}_{2} / \mathrm{WSe}_{2}$ heterostructures. 31 33. The collection of the photoexcited carriers can be enhanced by sandwiching $\mathrm{MoS}_{2} / \mathrm{WSe}_{2}$ between graphene 32]. Furthermore, Withers et al. demonstrated a new type of light-emitting diode based on van der Waals heterostructures, which consist of graphene, hexagonal boron nitride and various semiconducting TMCs such as $\mathrm{MoS}_{2}$. 34] The devices showed a quantum efficiency up to $8.4 \%$, and the emission spectrum can be tuned by choosing different semiconducting layers.

Furthermore, excitonic properties of 2D crystal structures show significant deviations from their bulk forms. While the exciton binding energy varies between few to $100 \mathrm{meV}$ in an usual semiconductor, 2D TMDs have exciton binding energies of several hundred meV. 22, 35 37. Such a strong enhancement in the excitonic binding energy is attributed to two main effects: (i) the confinement forces that localize the electron and the hole at the same place (which causes a stronger binding energy), and (ii) the dielectric screening of the Coulomb interaction is significantly reduced due to the dielectric mismatch effect as compared to the bulk. Additionally, it was demonstrated that absorption of a photon having energy larger than the bandgap in a $2 \mathrm{D}$ semiconductor can also led to the formation of biexcitons and trions (negatively charged electronexciton or positively charged hole-exciton). 25, 38 [43] As experimentally shown by Lin et al. [44]] (see Figure [4(b)) for $2 \mathrm{D}$ materials that have high surface-to-volume ratio the distribution of the Coulomb interaction and therefore the behavior of excitons are strongly modified by the dielectric environment. It was observed that different dielectric environments can tune the binding energies of excitons and trions by one order of magnitude. The relative binding energy of exciton and trion of the single-layer $\mathrm{MoS}_{2}$ are larger when the system is in vacuum and they decrease with the effective dielectric constant as shown in Figure 4(d). In another recent study the screening properties of two-dimensional semiconductors and layered structures were investigated by Latini et al. It was shown that while the screened interaction in the generalized Mott-Wannier model yields results almost identical to those of the strict 2D model (ab-initio calculations) for exciton binding energies, it may fail for heterostructures and when supported 2D materials are present. They also showed that a quasi-2D model, that takes into account the finite extension of the 2D material in the out-of-plane direction, provides a much broader applicability. 45]

Following the above-mentioned experimental works on the characterization of the optical properties of ultrathin crystals, we will focus here on the theoretical efforts 


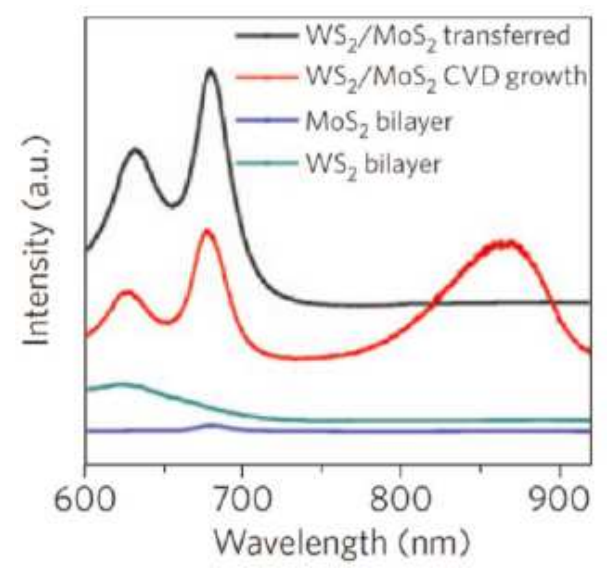

FIG. 3. PL spectrum of a CVD-grown $\mathrm{WS}_{2} / \mathrm{MoS}_{2}$ bilayer made by mechanical transfer, and CVD-grown $\mathrm{MoS}_{2}$ and $\mathrm{WS}_{2}$ bilayers [from Ref. 28].

and recent findings on the optical properties of two dimensional atomic-thin materials. Next section includes a detailed overview of the optical properties of: (i) elemental monolayers of group IV and V, (ii) derivatives of graphene, (iii) transition-metal chalcogenides, (iv) III-V binary compounds, and (v) heterostructures.

\section{THEORETICAL DETERMINATION OF OPTICAL PROPERTIES OF ULTRA-THIN CRYSTALS}

Despite the success of LDA [46] and GGA [47, 48] approximations in describing the exchange and correlation interactions and resulting electronic band dispersion of structures, electronic and optical energy bandgap values are mostly underestimated by DFT. Since simple Kohn-Sham eigenvalues give inaccurate quasiparticle energy spectrum for the structure, Kohn-Sham DFT can not explain properly the phenomena which include electronic excitations like photoemission or photoabsorption since the Kohn-Sham. 49] However, quite reliable results can be obtained by using beyond-DFT approaches such as HSE, GW and BSE.

One of the deficiencies of DFT arises from local exchange functionals, that give smaller ionization potential and electron affinity values. To solve this systematical error one can use hybrid functionals such as HSE; the short range part of the exchange term is defined as a mixture of exact short-range exchange from the Fock theory and semilocal long-range exchange of GGA. It was shown that nonlocal potetntials generated by HSE functionals accurately describe the band gap of semiconductos [50].

Although the GGA+HSE and LDA+HSE methods are successful in finding electronic bandgap, inclusion of the self energy of the electron $(i \mathrm{GW})$ is essential for cases in which the screening effects are large. This can be done by using the GW approximation 51 54 where G and W stand for the single particle Green's function and the screened Coulomb interaction, respectively. Briefly, the self energy of a particle is the energy which originates from the disturbance on the environment by the particle itself, and the particle with its disturbance is the quasiparticle $(\mathrm{QP})$. There are several methods to calculate the QP energies, such as the $\mathrm{G}_{0} \mathrm{~W}_{0}$ (single shot for the $\mathrm{G}$ and $\mathrm{W}$ ), $\mathrm{GW}_{0}$ (several iterations over the $\mathrm{G}$ and no iterations over the W), GW (several iterations over the G and W) as well as the scGW (fully self-consistent). These methods are implemented in softwares ABINIT, [5. Yambo, 56. QuantumEspresso, 57] and VASP. 58 61.

The inclusion of screening effects is sufficient to obtain the accurate electronic structure of a material. However, to obtain accurate optical spectrum, such as absorption and photoluminescence, the excitonic states must be taken into account. Because, in the optical excitation mechanism, an electron usually makes a bound state with a hole rather than moving back-and-forth between the QP states. Furthermore, considering only the excitations between the QP states underestimates the strength of the low energy excitations and overestimates the high energy excitations. Therefore, the Bethe-Salpeter equation (BSE) [62, 63] must be solved for the two-particle Green's function of electron-hole pairs. The BSE is expressed in terms of the wave functions and energies that are obtained from the QP calculation. The commonly used program packages which are mentioned above are also capable to solve the BSE.

\section{A. Elemental Monolayers of Group IV and V}

The pioneer of the 2D materials is graphene which is an elemental monolayer structure of carbon atoms. Beside its unusual mechanical and electronic properties, graphene displays many interesting optical features, such as a constant optical conductivity and tunable optical absorbance. After the synthesis of graphene in 2004, the first theoretical attempt to understand graphene's optical properties within the first-principles method was made by Yang et al. in 2009. They investigated the optical properties of single and bilayer graphene including many-electron effects. 64 It is commonly believed that excitonic effects are not important for metals because of the high screening. However, they reported that the excitonic effects significantly change the absorption spectrum (where the main peak position is red-shifted) in the energy regime near the van Hove singularity shown in Figure 5, whereas excitonic effects do not chance the absorbance in the infrared regime. 64 Trevisanutto et al. presented $a b$ initio many-body calculations of the optical absorption of single and bilayer graphene by solving the Bethe-Salpeter equation (BSE) on top of a GW approximation. For graphene, they predicted an excitonic resonance at $8.3 \mathrm{eV}$ arising from a background continuum of dipole forbidden transitions, and for the bilayer system, the resonance was predicted to be shifted to 9.6 

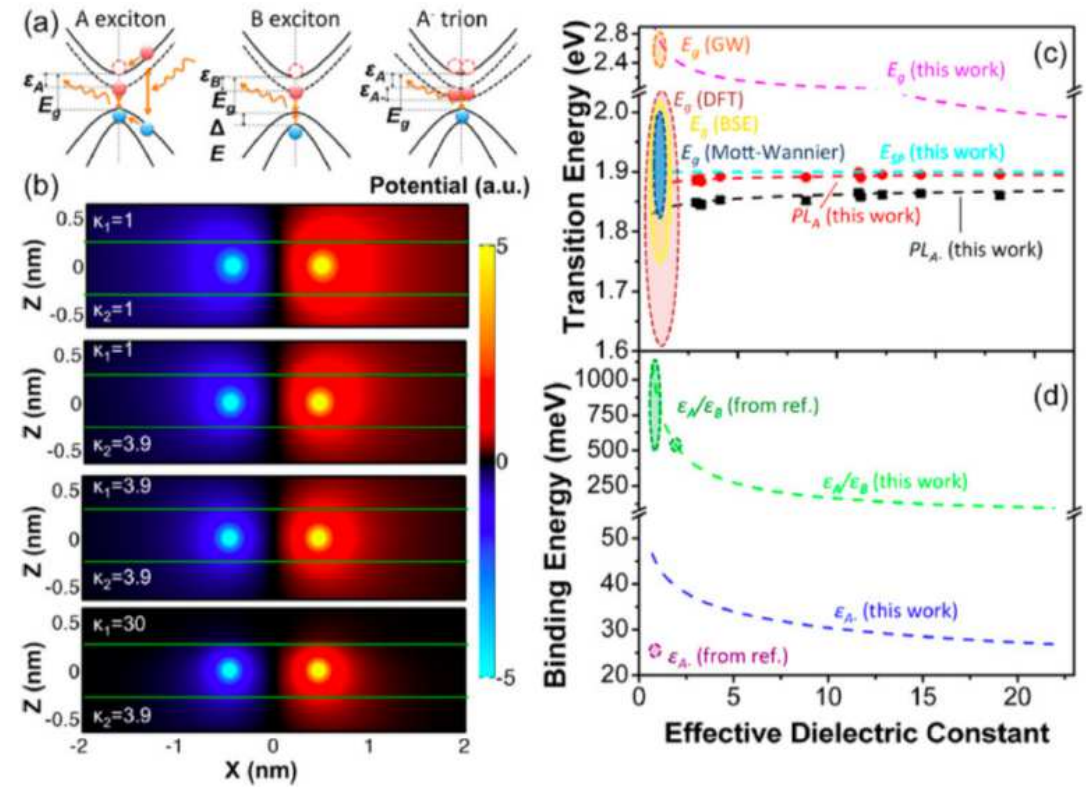

FIG. 4. (a) The radiative transitions related to excitons at the $K$-point in the BZ. $\mathrm{E}_{g}, \Delta, \epsilon_{A}, \epsilon_{B}, \epsilon_{A^{-}}$correspond to band gap, valence band splitting, binding energy of $\mathrm{A}, \mathrm{B}$, exciton and $\mathrm{A}^{-}$trion, respectively. (b) Contour plots of the Coulomb potential

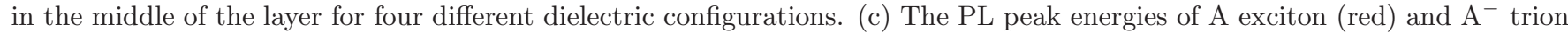
(black) as a function of the effective dielectric constant. (d) The binding energies of $\mathrm{A}, \mathrm{B}$ exciton (green) and $\mathrm{A}^{-}$trion (blue) as function of the effective dielectric constant [from Ref. 44].

\section{$\mathrm{eV} \cdot 65$}

The stacking-dependent optical spectrum of bilayer graphene, particularly twisted bilayer graphene, was calculated by Chen et al. using the first-principles GW-BSE approach. They demonstrated that electron-electron and electron-hole interactions must be included in the calculations of the optical spectrum. [66] The excitonic effects in twisted bilayer graphane with various twist angle was discussed in the first-principles GW-BSE scheme by Havener et al. and they showed that including electronhole interactions was essential to reproduce the conductivity spectrum. 67] They also found coherent interactions between states associated with multiple van Hove singularities in twisted bilayer graphene. Significance of the stacking order in few-layer graphene was investigated by Yan et al..68] Their results suggested that when compared with AB-stacked few-layer graphene ABC-stacking has significant effects on the optical absorption spectrum with an absorption peak that appeared around $0.3 \mathrm{eV}$. As the number of layers increases, the absorption amplitude is greatly enhanced, and the absorption peak redshifts.

Yang et al. performed first-principles calculations on graphene and bilayer graphene and reported that the high-frequency optical spectrum was dominated by both broad and narrow resonant excitons. Yang also identified the binding energy of this exciton; $270 \mathrm{meV}$ in graphene and $80 \mathrm{meV}$ in bilayer graphene. 69] Measurement of Mak et al. indicated that strong electron-hole interactions appeared in the optical conductivity at 4.62 $\mathrm{eV}$, which is redshifted by nearly $600 \mathrm{meV}$ from the value predicted by $a b$ initio GW calculations which is shown in
Figure 6, 70] This observation is explained within a phenomenological model as a Fano interference effect where the parameters for that model were extracted from experimental measurements. 71, 72] The Fano model was also used to describe the absorption of single- and bilayer graphene by Chae et al.. [73] Wachsmuth et al. performed first-principles calculations in the framework of timedependent DFT (TDDFT) and calculated the energy-loss function of single-layer graphene. Despite the disagreement with the experiment in the limit of small and vanishing momentum transfers, good agreement was found for finite momentum transfers if crystal local-field effects were taken into account. 74.

Effects of mechanical and chemical treatments on the optical properties of graphene were also investigated. First-principles calculations were performed to obtain electric field induced band gap and optical properties of bilayer graphene and suggested that optical absorbance is found to be strongly dependent on the stacking order of bilayer graphene, and the polarization direction of the incident light. 75, 76] A DFT calculation was performed within the $a b$ initio spin-density functional code AIMPRO [7] by Pereira et al. in order to find the optical conductivity of graphene under strain. 78. They reported that if the system is anisotropically strained, the optical response of graphene becomes frequency-independent. Yang et al. performed first-principles calculations on the optical absorption spectrum of doped graphene to reveal many-body effects. [79] These calculations showed that charge doping enhances the screening and reduces the e$\mathrm{h}$ interaction, as a result, the red shift of the absorbance 

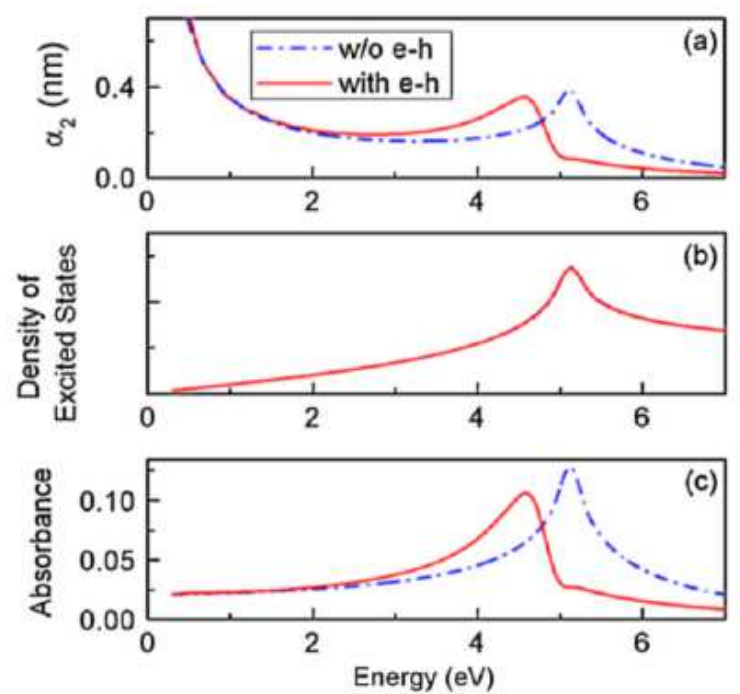

FIG. 5. (a) Optical absorption spectrum, (b) density of excited states, (c) absorbance of graphene, with and without excitonic effects included [from Ref. 64].

peak around $4.5 \mathrm{eV}$ is reduced. At different doping levels, the energy loss function of graphene at the K Dirac cone were calculated within DFT and the changes of lowenergy excitations around the Dirac cone with the carrier density was explained. [80] First-principles TDDFT formalism was also used to obtain the excitations near the Dirac cone for doped graphene by Despoja et al. 81] and it was reported that their findings agreed with experimental results.

Kadi et al. calculated the optical properties of Bernalstacked bilayer graphene using a density matrix formalism which includes fully momentum-dependent optical and Coulomb matrix elements. A noticeable peak is observed in the low energy spectrum of the structure which results from the cross transitions at the Dirac point. On the other hand, the spectrum in the ultraviolet region showed two close peaks originating from interband transitions. 82

Not only doping but also mechanical treatment is capable to tune the optical properties. Singh et al. studied the optical properties of clean and oxidized vacancies in graphene by means of DFT and indicated that some type of vacancies showed particular peaks in the absorption spectrum and it is possible to obtain the oxidation level from optical spectroscopy. [83] First-principles calculations of the dielectric function in the framework of the random-phase approximation (RPA) was carried out by Sedelnikova et al. and showed that out-of-plane bending of graphene removes the restriction on the absorption of certain light polarization, as a result, the energy position, intensity, and width of the peaks of the absorption curves are sensitive to bending. 84

Graphene nanoribbons (GNR), with different edge formations, are novel materials. Because of reduced dimensionality, excitonic effects are dominant in the op- tical spectrum and the binding energy of excitons is also large for ribbons which is of importance for optical applications. 85 87] Graphene nanoflakes with different shapes were also investigated. According to DFT calculations, nanoflakes, with van Hove singularities and edge states, showed a different optical spectrum in visible light. 88, 89] In addition to nanoribbons and nanoflakes, the synthesized stable 2D carbon allotrope graphdiyne, where the benzene rings are connected by acetylene bonds, is dominated by excitonic effects. This was calculated within the GW-BSE and was found to be a candidate for optical applications. [90, 91]

Silicene and germanene are 2D buckled hexagonal structures constructed from group-IV elements ( $\mathrm{Si}$ and Ge, respectively). Structural and electronic similarities with graphene make these materials of interest for their optical properties. Bechstedt et al. studied the optical absorbance of graphene, silicene, and germanene by ab-initio calculations within the independent-particle approximation. The low energy absorbance was found to be the same for all structures as predicted for massless Dirac fermions $(A(0)=\pi \alpha)$ and showed that the peaks and shoulders in the absorption spectrum can almost be related to band edges which are shown in Figure 7, 92, 93.

Cakir et al. calculated the optical properties of single layer black phosphorus (phosphorene) using GW-BSE method and found that the optical response of the monolayer strongly depends on the direction and the magnitude of the applied strain. [94] It was also demonstrated that the stacking of layers and the type of stacking modifies the optical properties of multilayer phosphorene. 95] Seixas et al. calculated the exciton binding energy of phosphorene under strain and found that strain modifies the binding energy of the excitons in the monolayer. 96 The optical response of phosphorene nanoribons was calculated by Tran et al. and they showed that although armchair phosphorene nanoribons have optical transitions close to the band gap, zigzag phosphorene nanoribons have symmetry-gap-forbidden transitions at the band edge. 97]

\section{B. Derivatives of Graphene}

Graphene can be modified by attaching hydrogen or halogen atoms. Such graphene derivatives gathered attention over the past years. Graphane is constructed by hydrogenation of the $\mathrm{C}$ atoms alternatingly above and below the graphene layer. Effect of hydrogenation of graphene on the optical spectrum was calculated in the independent particle approach of DFT by Pulci et al. They reported that the optical spectrum is dramatically changed. The electronic band gap of graphane is $3.5 \mathrm{eV}$ while the absorption curve is almost zero up to $7 \mathrm{eV}$. 98] Cudazzo et al. performed first principles calculations by solving the GW-BSE for graphane and demonstrated that the localized charge-transfer excitations, which were governed by enhanced electron correlations, dominated 


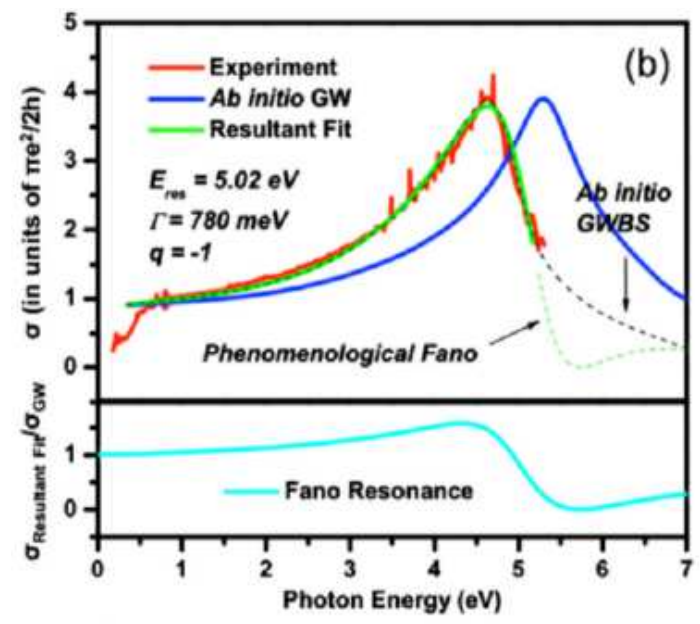

FIG. 6. Fit of experiment to the Fano model [from Ref. 70] using the optical conductivity obtained from GW calculations 64] for $\sigma_{\text {cont }}$. The dashed line is the optical conductivity spectrum obtained from the full GW-BSE calculation. 64]

the optical properties of graphane. 99] Wei et al. carried out first principles calculations within the GW-BSE scheme to obtain the optical properties of $\mathrm{C}_{4} \mathrm{H}$, which is partially hydrogenated graphene and they indicated that, as in ordinary graphane, strong charge-transfer excitonic effects dominated the optical properties, and the binding energy of the first exciton peak was found to be $1.67 \mathrm{eV}$. 100 Cheng et al. 101] focused on half-graphane, where only one sublattice was passivated by hydrogen, and investigated also $\mathrm{C}_{6} \mathrm{H}$ to obtain the electronic and the optical properties by using the ABINIT code 55$]$. They reported that the hydrogen atom behaves as an impurity, and this caused an enhancement of the optical conductivity.

The optical response of fluorographene was investigated by Samarakoon et al. using the GW-BSE approach and they reported that the results were in good agreement with the experiments. 102] Charge-transfer excitations arising from strong electron-hole interactions were obtained. In addition, Karlický et al. calculated the optical properties of chlorographene $(\mathrm{CCl})$, fluorographene $(\mathrm{CF})$, and graphane $(\mathrm{CH})$ at the GW-BSE level and found the formation of exciton peaks at 2.82, 5.12, and $4.11 \mathrm{eV}$ with huge binding energies of $1.25,1.85$, and 1.53 $\mathrm{eV}$ for $\mathrm{CCl}, \mathrm{CF}$, and $\mathrm{CH}$, respectively, as shown in Figure 8, 103] The importance of excitonic interaction for single-, double-layer and bulk CF was also studied by Karlický et al. and they reported additionally that the dominant high-energy peak of excitonic absorption systematically shifted to higher energies by increasing the number of layers which can be useful for the determination of the thickness of samples. [104] In addition, Wei et $a l$. reported that the inclusion of excitonic effects is crucial for the determination of the optical properties of $\mathrm{CF}$ and $\mathrm{C}_{4} \mathrm{~F}$ after performing a first-principle calculation at
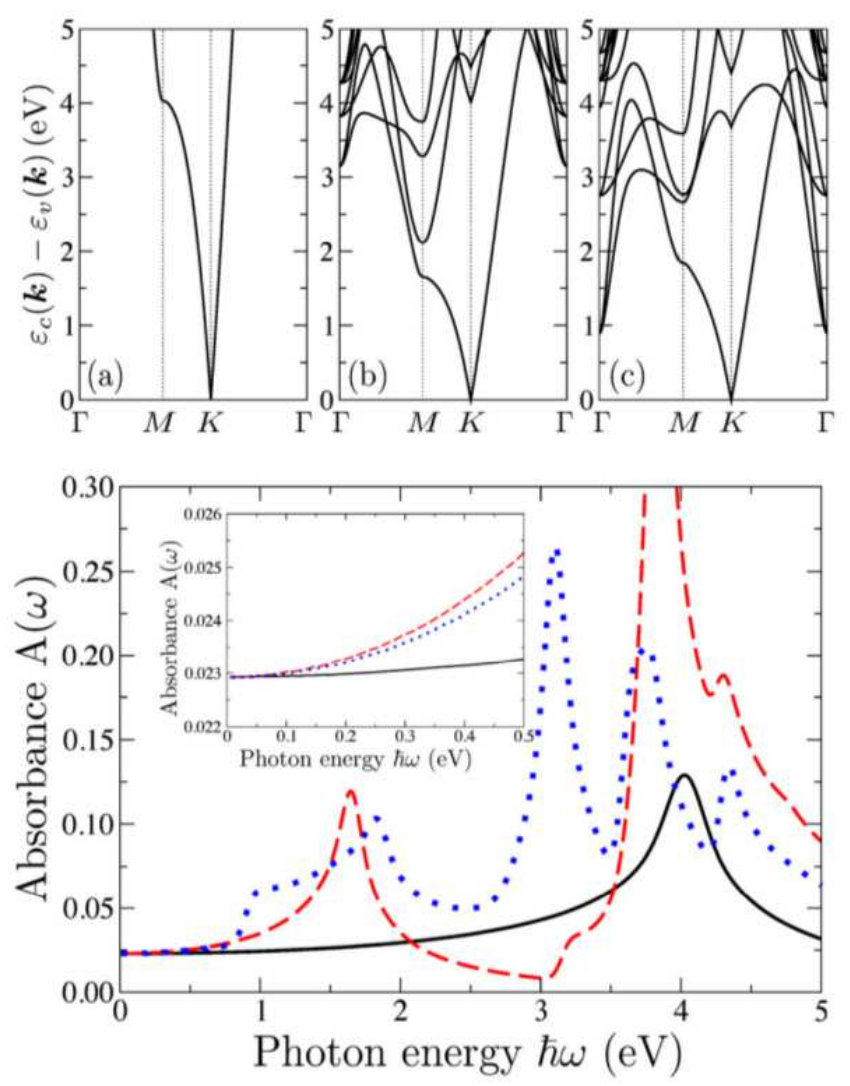

FIG. 7. Interband transition energies along high-symmetry lines in the Brillouin zone (BZ) for graphene (a), silicene (b), and germanene (c). Lower panel, ab-initio calculated optical absorbance of graphene (black solid line), silicene (red dashed line), and germanene (blue dotted line) vs photon energy. The infrared absorbance is shown in the inset [from Ref. 92].

the level of GW-RPA and GW-BSE. 105] Moreover, Gunasinghe et al. [106] presented DFT calculations within the GW-BSE approach as implemented in the YAMBO package. 56. It was also indicated that the results of GWBSE were in good agreement with the experiment, and inclusion of the e-h interactions is important for description for the excitation effect.

\section{Transition-Metal Chalcogenides}

Due to potential applications in the fields of optoelectronics and photonics of single layer transition-metal dichalcogenides (TMDs) there has been an immense interest in the investigation of their optical properties. Since they are expected to have strong excitonic effects due to the weak dielectric screening which will strongly influence their optical properties, it is vital to include these effects into the calculations.

$\mathrm{MoS}_{2}$ is one of the most investigated 2D materials among the TMDs. It is an indirect gap semiconductor in its bulk form with a band gap of around $1 \mathrm{eV}$ [107] and 

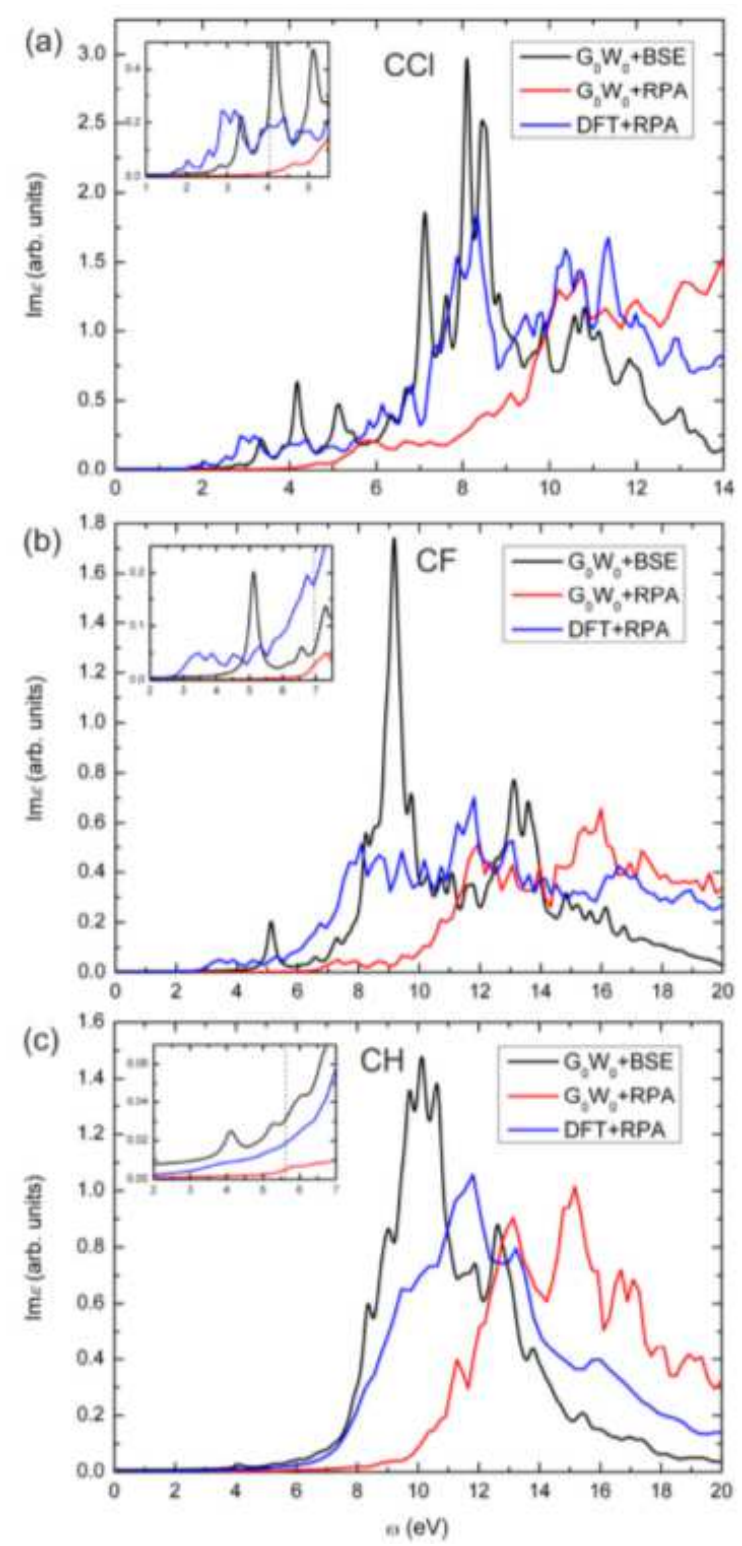

FIG. 8. Absorption spectrum of monolayer (a) $\mathrm{CCl}$, (b) $\mathrm{CF}$, and (c) $\mathrm{CH}$ for light polarization parallel to the surface plane. Insets show amplified regions of the absorption spectrum in the vicinity of the first exciton peak and $\mathrm{G}_{0} \mathrm{~W}_{0}$ (PBE) gap (dashed line) [from Ref. 103].

it becomes a direct gap semiconductor when it is isolated as a monolayer. [17, 108] Monolayer TMDs and particularly $\mathrm{MoS}_{2}$ is a challenging system for calculating the optical properties because the results are quite sensitive to the settings such as the number of empty bands $\left(\mathrm{N}_{b}\right)$, the energy cutoff for the response function $\left(\mathrm{E}_{r}\right)$ and the sampling of the Brillouin zone used in the calculations. That is why different settings lead to different results for the optical spectrum. It has been shown that the dependence of the optical spectrum on the calculation settings are more prominent when the quasiparticle wave functions contain localized states and the conduction band minimum $(\mathrm{CBM})$ and valence band maximum (VBM) have different orbital character. 109] It is known that the VBM of monolayer $\mathrm{MoS}_{2}$ at $\mathrm{K}$ and $\mathrm{K}^{\prime}$ is strongly delocalized by the $d_{x^{2}-y^{2}}$ orbital from the Mo atom, and the $p_{x}$ and $p_{y}$ orbitals from the $\mathrm{S}$ atom. On the other hand, the CBM has $d_{z^{2}}$ character from the Mo atom and a small contribution from $p_{x}$ and $p_{y}$ orbitals of the $\mathrm{S}$ atom. 223] This makes the system computationally challenging.

It is known from experiments that the positions of the $\mathrm{A}$ and $\mathrm{B}$ exciton peaks in $\mathrm{MoS}_{2}$ monolayer are around $1.85 \mathrm{eV}$ and $1.98 \mathrm{eV}$, respectively. Band structure calculations show that this splitting is due to the effect of spin-orbital coupling (SOC) at the $\mathrm{K}$ point of the BZ. It was also found in the literature that the photoluminescence peak increases from bulk to monolayer $\mathrm{MoS}_{2}$, which is consistent with the indirect-to-direct band gap crossover as the sample is reduced to a monolayer.

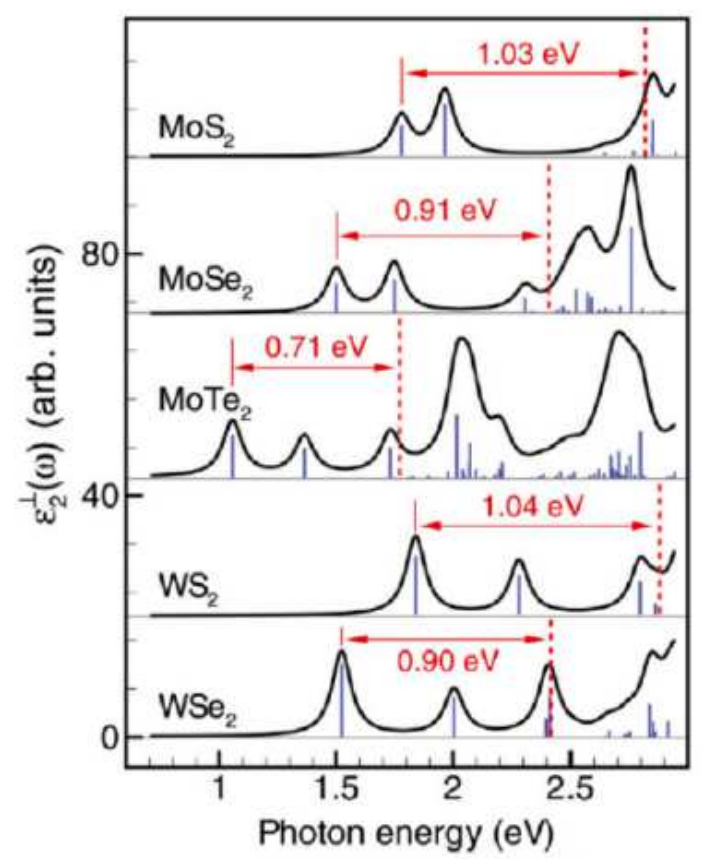

FIG. 9. Imaginary part of the dielectric constant for monolayer (a) $\mathrm{MoS}_{2}$, (b) $\mathrm{MoSe}_{2}$, (c) $\mathrm{MoTe}_{2}$, (d) $\mathrm{WS}_{2}$, and $\mathrm{WSe}_{2}$. The blue lines correspond to the relative oscillator strengths for the optical transitions. The dashed red lines indicate the $\mathrm{G}_{0} \mathrm{~W}_{0}$ band gap. The binding energy of A exciton is indicated in the figure [from Ref. 111]).

In the literature there are several works on the absorption spectrum of monolayer $\mathrm{MoS}_{2}$. Cheiwchanchamnangij et al. showed that by using the self consistent GW method with Mott-Wannier theory, the $\mathrm{MoS}_{2}$ is a direct gap semiconductor. [110] The calculation was performed with a k-mesh of $8 \times 8 \times 2$ for monolayer $\mathrm{MoS}_{2}$ and it was found that the transition energy values for $\mathrm{A}$ and $\mathrm{B}$ excitons are 2.75 and $2.9 \mathrm{eV}$ which is slightly overestimated as compared to the experimental values. Similarly, Ramasubramaniam showed that the $\mathrm{MoS}_{2}$ is also an direct gap semiconductor by using the single-shot GW method 
TABLE I. The positions of A, B, A', B', and C exciton peaks from experiments and several theoretical calculations. The convergence criterion i.e. used k mesh, number of valence $\left(\mathrm{N}_{v}\right)$ and conduction bands $\left(\mathrm{N}_{c}\right)$, the energy cutoff for the response function $\left(\mathrm{E}_{r}\right)$ and the number of empty bands $\left(\mathrm{N}_{b}\right)$ used in the calculations are also listed.

\begin{tabular}{|c|c|c|c|c|c|c|c|c|c|c|c|c|}
\hline & \multicolumn{7}{|c|}{ Optical Transition (eV) } & \multicolumn{5}{|c|}{ Convergence Parameters } \\
\hline & A & B & $\mathrm{A}^{\prime}$ & $\mathrm{B}^{\prime}$ & $\mathrm{C}$ & $\mathrm{E}_{b}$ & $\mathrm{E}_{g} \mathrm{GW}$ & k-mesh & $\mathrm{N}_{v}$ & $\mathrm{~N}_{c}$ & $\mathrm{E}_{r}(\mathrm{eV})$ & $\mathrm{N}_{b}$ \\
\hline Absorp. Exp.[108] & 1.88 & 2.03 & - & - & - & - & - & - & - & - & - & - \\
\hline PL Exp.[17] & 1.85 & 1.98 & - & - & - & - & - & - & - & - & - & - \\
\hline $\mathrm{G}_{0} \mathrm{~W}_{0}-\mathrm{BSE}[111]$ & 1.78 & 1.96 & - & - & - & 1.04 & 2.82 & $6 \times 6 \times 1$ & 2 & 4 & 272 & 96 \\
\hline sc-GW $\mathrm{G}_{0}-\mathrm{BSE}[112]$ & 2.22 & 2.22 & 2.50 & 2.50 & - & 0.63 & 2.80 & $15 \times 15 \times 1$ & 6 & 8 & 300 & 197 \\
\hline $\mathrm{G}_{1} \mathrm{~W}_{0}-\mathrm{BSE}[114]$ & 1.88 & 2.02 & 2.20 & 2.32 & 2.54 & 0.96 & 2.84 & $72 \times 72 \times 1$ & 7 & 8 & 476 & 6000 \\
\hline
\end{tabular}

$\left(\mathrm{G}_{0} \mathrm{~W}_{0}\right)$ on top of Heyd-Scuseria-Ernzerhof (HSE) hybrid calculations which is shown in Figure 9, 111] The calculation was performed with a $6 \times 6 \times 1 \mathrm{k}$-mesh, $272 \mathrm{eV}$ for the $\mathrm{E}_{r}$ and the $\mathrm{N}_{b}$ value was taken as 96.2 highest valence bands $\left(\mathrm{N}_{v}\right)$ and 4 lowest conduction bands $\left(\mathrm{N}_{c}\right)$ were included as basis for the excitonic state at the BSE level. With these settings the positions of $\mathrm{A}$ and $\mathrm{B}$ excitons were found to be quite similar to the experimental values, 1.78 and $1.96 \mathrm{eV}$, respectively and with an exciton binding energy of $1.04 \mathrm{eV}$ (see Table 【). By using self-consistent GW method, Shi et al. showed that the monolayer $\mathrm{MoS}_{2}$ is an indirect band gap semiconductor at the $\mathrm{G}_{0} \mathrm{~W}_{0}$ level and updating the $\mathrm{G}$ part makes it a direct band gap semiconductor. 112] In this calculations a $15 \times 15 \times 1 \mathrm{k}$-mesh is used together with $300 \mathrm{eV}$ for $\mathrm{E}_{r}, \mathrm{~N}_{b}$ value is taken as 197 and the $\mathrm{N}_{v}$ and $\mathrm{N}_{c}$ are selected as 6 and 8 , respectively. In this calculation in addition to $\mathrm{A}$ and $\mathrm{B}$ exciton peaks (at $2.22 \mathrm{eV}$ ), the $\mathrm{A}^{\prime}$ and $\mathrm{B}^{\prime}$ exciton peaks (at $2.50 \mathrm{eV}$ ) are also found which are not experimentally active (see Table \). The exciton binding energy was calculated as $0.63 \mathrm{eV}$ in this study. Noticed that results exhibit clear discrepancies due to the different settings used in the calculations. For instance the dependence of the optical spectrum of the monolayer $\mathrm{MoS}_{2}$ on the Brillouin zone sampling is shown in Figure 10. As can be seen the position of the peaks are moving towards higher energy values with increasing the k-point grid. 112, 113.

In order to address these discrepancies, a more recent work by Qiu et al. calculated the absorption spectrum of monolayer $\mathrm{MoS}_{2}$ with very high settings. [114] In this study the G part is updated self-consistently one time $\left(\mathrm{G}_{1} \mathrm{~W}_{0}\right)$ because an additional iteretion change the the band gap by less then $20 \mathrm{meV}$. A $72 \times 72 \times 1 \mathrm{k}$-mesh is used with energy cutoff of $\mathrm{E}_{r}=476 \mathrm{eV}$ and $\mathrm{N}_{b}=$ 6000 bands where both parameters must be converged together for the accurate QP energies. The number of valance bands $\mathrm{N}_{v}$ and conduction bands $\mathrm{N}_{c}$ are selected as 7 and 8 for BSE calculation, respectively. It was predicted that there are exciton peaks in the 2.2 and 2.8 $\mathrm{eV}$ range. These peaks were not found in other theoretical and experimental works. Calculated absorption spectrum is shown in Figure 11. They suggested that the previous calculations [111, 112] were not converged with respect to k-point sampling, and the agreement be-

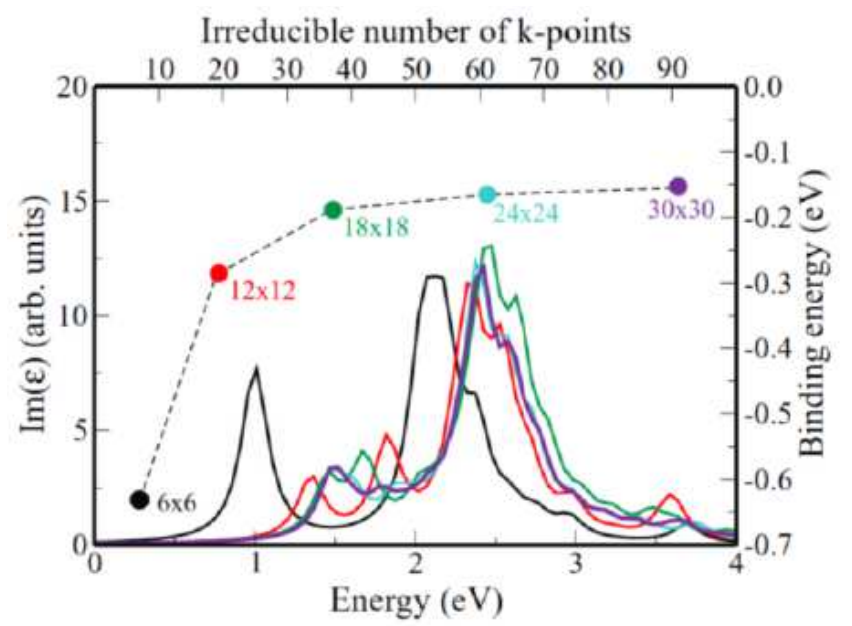

FIG. 10. Imaginary part of the dielectric function, obtained by solving BSE, of $\mathrm{MoS}_{2}$ for several k-point meshes (Left axis). Binding energy of the exciton $\left(\mathrm{E}_{b}\right)$ as a function of the number of irreducible k-points (Right axis) [from Ref. 113].

tween the peak position for A and B and experimental results found in earlier reports are likely accidental. It was also suggested by Qiu et al. that the inconsitancy between the converged calculations and the experiment can be solved by considering the quasiparticle lifetime effect due to electron-phonon interaction which is shown in Figure 11 (b).

In addition to $\mathrm{MoS}_{2}$, Ramasubramaniam et al. calculated the optical properties of $\mathrm{MoSe}_{2}, \mathrm{MoTe}_{2}, \mathrm{WS}_{2}$, $\mathrm{WSe}_{2}$ and $\mathrm{WTe}_{2}$ solving BSE on top of $\mathrm{G}_{0} \mathrm{~W}_{0}$ and $\mathrm{HSE}$ hybrid functional method. It was reported that these materials have strongly bound excitons which have binding energies around $1 \mathrm{eV}$. These binding energies are becoming smaller and exciton peaks show a systematic redshift as the chalcogen atoms get heavier. [111] The absorption spectrum together with the oscillator strength (shown by blue lines) are shown in Figure 9. The height of the lines are a measure of the oscillator strength.

The binding energy of excitons in monolayer and bilayer $\mathrm{MoS}_{2}$ was also calculated by using Mott-Wannier theory and reported as $0.897 \mathrm{eV}$ and $0.424 \mathrm{eV}$, respectively. [110] As seen the exciton binding energy is smaller in bilayer $\mathrm{MoS}_{2}$. 


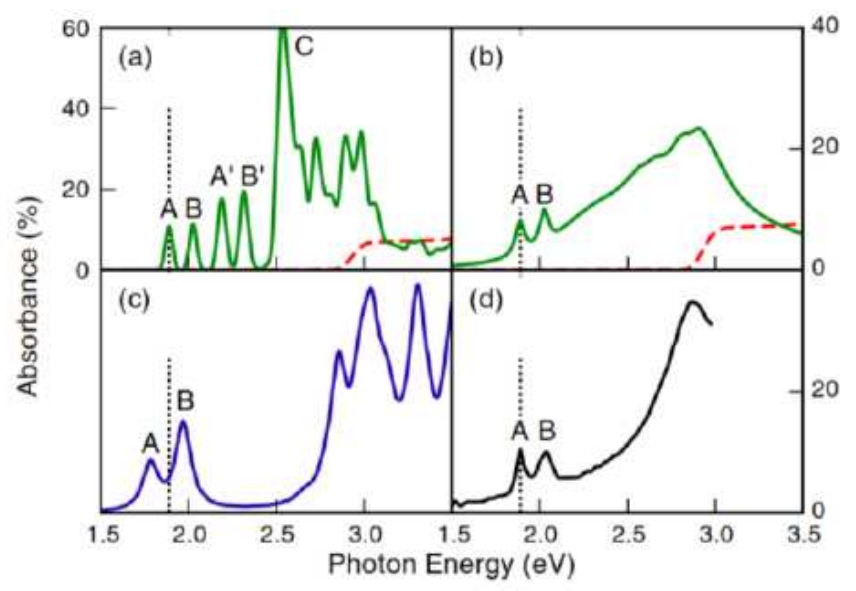

FIG. 11. (a) Absorption spectrum of $\mathrm{MoS}_{2}$ without (dashed red curve) and with (solid green curve) electron-hole interaction using a constant broadening of $20 \mathrm{meV}$. (b) Same data using an $a b$ initio broadening based on the electron-phonon interaction. (c) Previous calculation using $\mathrm{G}_{0} \mathrm{~W}_{0}$ (d) Experimental data [from Ref. 114].

The strain dependent optical absorption spectrum of single layer $\mathrm{MoS}_{2}$ was investigated by using BSE calculations on top of $\mathrm{G}_{0} \mathrm{~W}_{0}$ [115] and self-consistent $\mathrm{GW}_{0}$ [112] calculations. The reported binding energies of excitons are smaller than the previous values which are around $0.5 \mathrm{eV}$ and it was reported that these binding energies were not affected by biaxial strain.

The optical properties of other nanostructure forms of TMDs, such as $\mathrm{MoS}_{2}$ nanoflakes, were also investigated by using the density functional tight-binding (DFTB) method. It has been shown that with increasing edge length of the structures the absorption peaks are redshifted which is due to the quantum confinement effect. 116] Jing et al. showed that adsorbing organic molecules on $\mathrm{MoS}_{2}$ leads to a considerable charge transfer from the adsorbed molecule to the $\mathrm{MoS}_{2}$ layer. This transfer changes the electronic structure of the material but not much effect on the absorption spectrum of the system. [117]

The optical properties of low dimensional materials have are strongly correlated with the surrounding dielectric environment. In monolayer $\mathrm{MoS}_{2}$, this correlation leads to a blue shift up to $40 \mathrm{meV}$ in the photoluminescence (PL) peaks. Lin et al. analyzed this phenomena using an analytical investigation and showed that the surrounding dielectric environment has an effect on the exciton and trion binding energies of monolayer $\mathrm{MoS}_{2}$. 44

Vacancies and defects in TMD monolayers changes the optical properties. Wei et al. showed that atomic defects in $\mathrm{WS}_{2}$ monolayers lead to an increase in the absorption intensity and a red shift in the absorption spectrum of the material by using first-principle methods. [118] An analytical investigation showed the effect of point defects in $\mathrm{MoS}_{2}$ and $\mathrm{WS}_{2}$ monolayers on the optical properties of the materials. It was shown that the localized charge carriers around these point defects modify the optical characteristics of the systems. [119] Feng et al. showed that the static dielectric constant of monolayer $\mathrm{MoS}_{2}$ decrease with Mo vacancies and increase with $S$ vacancies by using first-principle calculations. 120] The stacking of these TMDs has also an influence on the optical spectrum of these materials. He et al. investigated this phenomena by using BSE calculations on top of $\mathrm{G}_{0} \mathrm{~W}_{0}$ with spin-orbit coupling for bilayer $\mathrm{MoS}_{2}, \mathrm{MoSe}_{2}, \mathrm{WS}_{2}$, and $\mathrm{WSe}_{2}$. They showed that stacking has an effect on the exciton binding energies of these systems. 121]

\section{Heterostructures made of Monolayer Building Blocks}

The optical properties of heterostructures which are made of $\mathrm{MX}_{2}$, graphene and hexagonal boron nitride monolayers are also of interest due to their unique optical properties. Even preliminary results on such materials as mentioned below have revealed the possibility of using heterostructures in various optoelectronics device applications.

The counterpart of graphene, $h$-BN is a well-known $2 \mathrm{D}$ crystal that differs from graphene with its insulating properties. Heterostructures of both materials are promising for not only electronic but also for advanced optical applications. Calculations of Yan et al. by including e-h interactions through the BSE-RPA showed that the absorption spectrum of the $h$-BN-on-graphene system is not simply a sum of the absorptions of the isolated layers which is shown in Figure 12, because the transition energies in $h$-BN become redshifted by $\sim 1 \mathrm{eV}$ due to the screening by the graphene electrons. [122] Attaccalite et al. focused on the effect of defects on the optical properties and reported that "deep level impurity" peaks in the optical spectrum are strongly affected by the e-e and e-h interaction and can be reliably calculated only on the level of many-body perturbation theory. [123] Wang et al. investigated the possible hyperlens application of graphene and $h$-BN by using DFT. 124] They reported that both graphene and $h$-BN exhibit strong anisotropic properties in the ultraviolet region, where their permittivity components perpendicular to the optical axis can be negative, while the components parallel to the optical axis can be positive, which is desirable for hyperlens implementations.

The optoelectronical properties of lateral hybridized graphene and hexagonal boron nitride was also investigated by solving BSE on top of GW calculations. [125] It is shown that the exciton binding energy decreases with increasing the carbon domain size in the system. The vertical heterostructure made of graphene and boron nitride (BN) was investigated by solving BSE and using functional by Gritsenko, Leeuwen, Lenthe, and Baerends (GLLBSC). It is predicted that the absorption spectrum of heterostructures is not equal to the sum of the individual absorption spectra. 

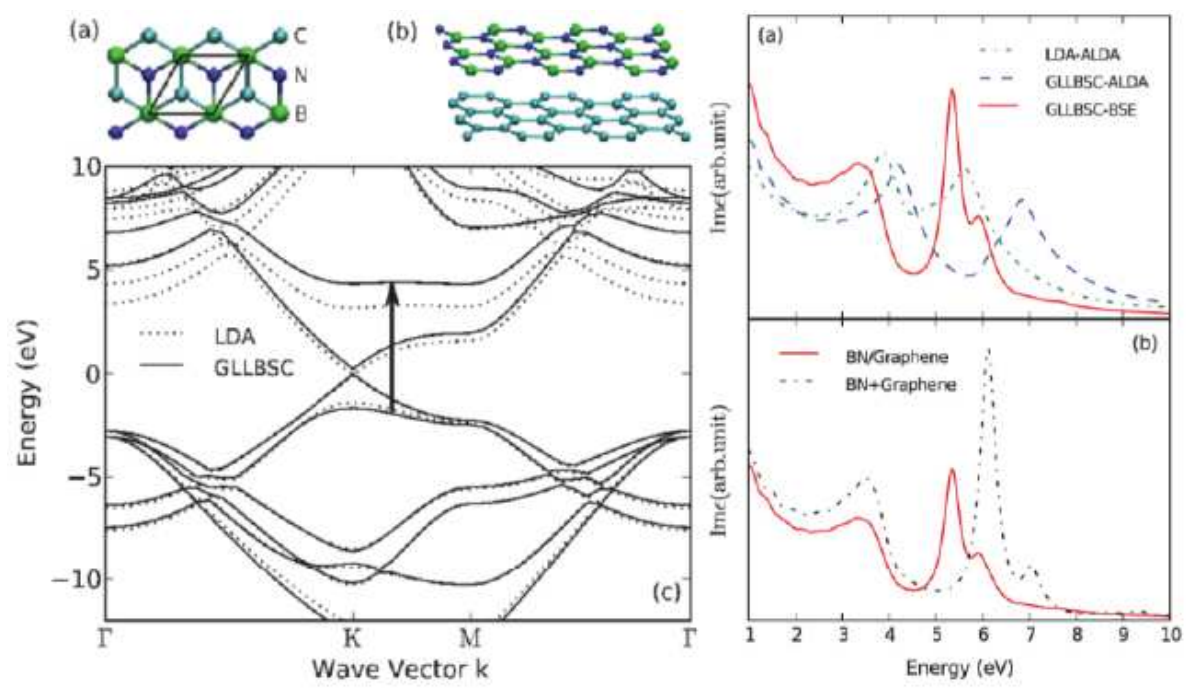

FIG. 12. (a) Top and (b) side view of a graphene/h- BN. (c) Band structure of graphene/h-BN calculated with GLLBSC (solid lines) and LDA (dotted lines). The top of the valence bands is set to zero. Upper right panel: Optical absorption spectrum of graphene/h-BN calculated using the LDA-ALDA (dash-dotted line), GLLBSC-ALDA (dashed line), and GLLBSC-BSE (solid line). Lower right panel: The GLLBSC-BSE spectrum of the interface (repeated) together with the sum of the absorption spectrum of an isolated graphene and h-BN layer, respectively [from Ref. 122].

However, heterostructures of TMDs provide another rich playground. Komsa et al. calculated the optical absorption spectrum of vertical $\mathrm{MoS}_{2} / \mathrm{WS}_{2}$ heterostructure by solving BSE equation on top of single shot $\mathrm{G}_{0} \mathrm{~W}_{0}$ calculation [126] and it was observed that the optical spectrum of such a heterostructure has the same features as the individual monolayers and the optically active transitions are formed by direct intralayer transitions.

Recently Kang et al. showed that certain van der Waals heterostructures, can facilitate spatial separate electron-hole localization which may find applications in photovoltaic energy conversion. [127] The electronic structure of various heterostructures were calculated in the GW level by Lebégue et al. They predicted that due to the band alignment, the $\mathrm{MX}_{2}$ heterostructures can be used as charge separator for solar cell applications. 128] The exciton radiative lifetimes of $\mathrm{MoS}_{2} / \mathrm{WS}_{2}$ and $\mathrm{MoSe}_{2} / \mathrm{WSe}_{2}$ bilayer heterostructures are calculated by solving the BSE equation on top of GW calculation. It is predicted that these heterobilayers exhibit long-lived $(\sim 20-30$ ns at room temperature) excitons whose electron is localized on the Mo and the holes are localized on the $\mathrm{W}$ based layer. 129]

Recent experiments on vdW heterostructures of TMDs have also revealed fingerprints of some unusual properties in their photoluminescence spectrum. In Figure 13. we present our first-principle calculations on $\mathrm{MoS}_{2}$ and $\mathrm{WSe}_{2}$ monolayers. As seen from the band structures both monolayers are direct band gap semiconductors. The band gap of the $\mathrm{MoS}_{2}$ monolayer is slightly larger than the band gap of the $\mathrm{WSe}_{2}$ monolayer and the splitting at the $\mathrm{K}$ point in the Brillouin zone is more apparent for $\mathrm{WSe}_{2}$ due to having a larger SOC. The band alignment calculations show that a possible heterostructure formed from these monolayers would be a type II heterojunction. This leads to spatially indirect excitons due to localization of the electrons and holes in different monolayers of the heterostructure. The recombination of the electron hole pair occurs through the staggered gap of the heterojunction. In Figure 13(d) and (e), the imaginary part of the dielectric function and oscillator strength of the optical transitions of $\mathrm{MoS}_{2}$ and $\mathrm{WSe}_{2}$ monolayers are shown, respectively. To obtain the dielectric function of these monolayers, BSE calculation on top of single shot GW calculation $\left(\mathrm{G}_{0} \mathrm{~W}_{0}\right)$ calculation is performed. Our calculations revealed that the oscillator strengths of the optical transition for $\mathrm{MoS}_{2}$ monolayer is smaller than the oscillator strengths of the $\mathrm{WSe}_{2}$ monolayer which fits with the earlier experimental findings performed by Fang et al. 130. In the same work it has been also shown that the oscillator strength of the optical transitions for $\mathrm{MoS}_{2}$ $\mathrm{WSe}_{2}$ heterostructure resembles the oscillator strength of the $\mathrm{MoS}_{2}$ monolayer. This shows that the oscillator strength of the heterojunction is dictated by the constituent monolayers which has lower oscillator strength.

Despite the increasing number of experimental studies on the optical properties of heterostructures, there are still few theoretical works due to the computational expense of such calculations.

\section{CONCLUSIONS}

In conclusion, advances in theoretical calculations on the optical properties of atomically thin layered crystal structures are reviewed. 2D crystals have been a topic of 

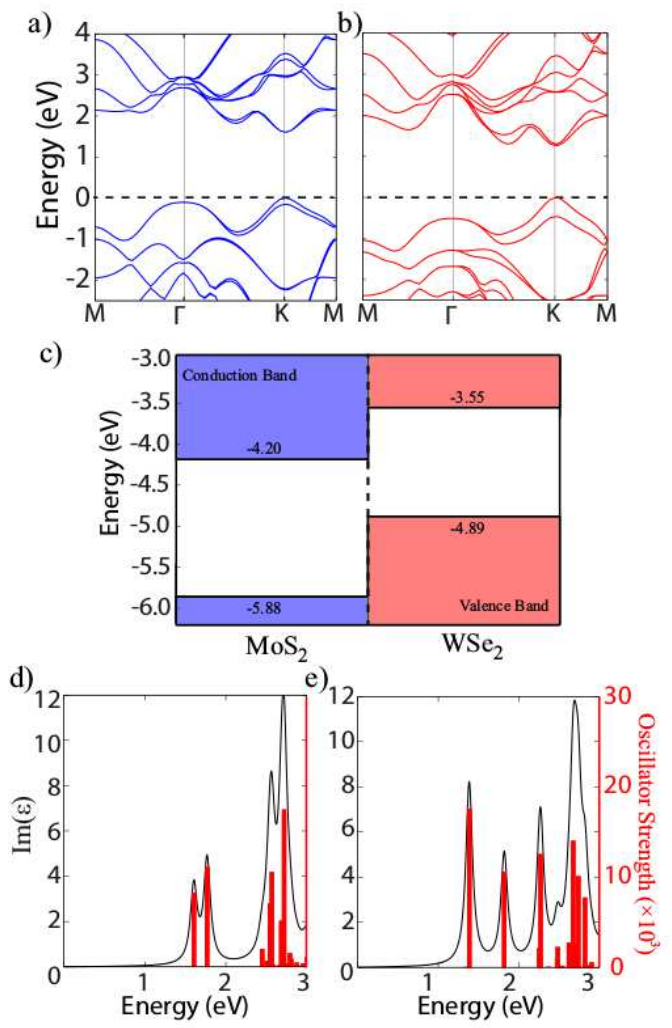

FIG. 13. The band structure of (a) $\mathrm{MoS}_{2}$ and (b) $\mathrm{WSe}_{2}$ with SOC, Fermi level is set to 0 eV. (c) Calculated band aligment for $\mathrm{MoS}_{2}$ and $\mathrm{WSe}_{2}$ monolayers. Imaginary part of the dielectric function for (d) $\mathrm{MoS}_{2}$ and (e) WSe 2 together with oscillator strenght of the optical transitions.

interest for more than a decade due to their unique properties which differ from their 3D counterparts. However, still significant research efforts are needed for deeper understanding of their unique optical properties. Regarding the difficulties in synthesis and characterization of materials at nanoscale, theoretical calculations that provides cheap, fast and reliable tool for analysis of ultra-thin crystal structures are of crucial importance. This overview reveals the power of theoretical predictions on the optical properties of graphenelike ultrathin crystal structures.

\section{ACKNOWLEDGMENTS}

This work was supported by the Flemish Science Foundation (FWO-Vl) and the Methusalem foundation of the Flemish government. Computational resources were provided by TUBITAK ULAKBIM, High Performance and Grid Computing Center (TR-Grid e-Infrastructure). H.S. is supported by a FWO Pegasus Long Marie Curie Fellowship. J.K. is supported by a FWO Pegasus short Marie Curie Fellowship.
[1] Novoselov KS, Geim AK, Morozov SV, Jiang D, Zhang Y, Dubonos SV, Grigorieva IV, Firsov AA. Electric Field Effect in Atomically Thin Carbon Films. Science 2004, 306:666-669.

[2] Geim AK, Grigorieva IV. Van der Waals heterostructures. Nature 2013, 499:419-425.

[3] Nair RR, Blake P, Grigorenko AN, Novoselov KS, Booth TJ, Stauber T, Peres NMR, Geim AK. Fine Structure Constant Defines Visual Transparency of Graphene. Science 2008, 320:1308.

[4] Mak KF, Sfeir MY, Wu Y, Lui CH, Misewich JA, Heinz TF. Measurement of the Optical Conductivity of Graphene. Phys Rev Lett 2008, 101:196405.
[5] Sun D, Wu Z-K, Divin C, Li X, Berger C, de Heer WA, First PN, Norri TB. Ultrafast Relaxation of Excited Dirac Fermions in Epitaxial Graphene Using Optical Differential Transmission Spectroscopy. Phys Rev Lett 2008, 101:157402.

[6] Wang F, Zhang Y, Tian C, Girit C, Zettl A, Crommie M, Shen YR. Gate-Variable Optical Transitions in Graphene. Science 2008, 320:206-209.

[7] Xia F, Mueller T, Lin Y-m, Valdes-Garcia A, Avouris P. Ultrafast graphene photodetector. Nat Nanotech 2009, 4:839-843.

[8] Pan D, Zhang J, Li Z, Wu M. Hydrothermal route for cutting graphene sheets into blue-luminescent graphene quantum dots. Adv Mater 2010, 22:734-738. 
[9] Jin SH, Kim DH, Jun GH, Hong SH, Jeon S. Tuning the Photoluminescence of Graphene Quantum Dots through the Charge Transfer Effect of Functional Groups. ACS Nano 2013, 7:1239-1245.

[10] Gokus T, Nair RR, Bonetti A, Bohmler M, Lombardo A, Novoselova KS, Geim AK, Ferrari AC, Hartschuh A. Making Graphene Luminescent by Oxygen Plasma Treatment. ACS Nano 2009, 3:3963-3968.

[11] Eda G, Lin Y-Y, Mattevi C, Yamaguchi H, Chen HA, Chen I-S, Chen C-W, Chhowalla M. Blue Photoluminescence from Chemically Derived Graphene Oxide. Adv Mater 2010, 22:505.

[12] Chien CT, Li SS, Lai WJ, Yeh YC, Chen HA, Chen IS, Chen LC, Chen KH, Nemoto T, Isoda S, Chen MW, Fujita T, Eda G, Yamaguchi H, Chhowalla M, Chen CW. Tunable photoluminescence from graphene oxide. Angew Chem Int Ed 2012, 51:6662-6666.

[13] Li Y, Chernikov A, Zhang X, Rigosi A, Hill HM, van der Zande AM, Chenet DA, Shih E-M, Hone J, Heinz TF. Measurement of the optical dielectric function of monolayer transition-metal dichalcogenides: $\mathrm{MoS}_{2}, \mathrm{MoSe}_{2}$, $\mathrm{WS}_{2}$, and $\mathrm{WSe}_{2}$. Phys Rev B 2014, 90:205422.

[14] Island JO, Buscema M, Barawi M, Clamagirand JM, Ares JR, Sánchez C, Ferrer IJ, Steele GA, van der Zant HS, Castellanos-Gomez A. Ultrahigh Photoresponse of Few-Layer $\mathrm{TiS}_{3}$ Nanoribbon Transistors. Adv Opt Mater 2014, 2:641-645.

[15] Barawi M, Flores E, Ferrer IJ, Ares JR, Sánchez C. Flat band potential of $\mathrm{TiS}_{3}$ in a photoelectrochemical cell is determined and the photogenerated hydrogen is quantified by mass spectrometry. J Mater Chem A 2015, 3:7959-7965

[16] Mak KF, Lee C, Hone J, Shan J, Heinz TF. Atomically Thin $\mathrm{MoS}_{2}$ : A New Direct-Gap Semiconductor. Phys Rev Lett 2010, 105:136805.

[17] Splendiani A, Sun L, Zhang Y, Li T, Kim J, Chim CY, Galli G, Wang F. Emerging Photoluminescence in Monolayer $\mathrm{MoS}_{2}$. Nano Lett 2010, 10:1271-1275.

[18] Tonndorf P, Schmidt R, BBöttger P, Zhang X, BBörner J, Liebig A, Albrecht A, Kloc C, Gordan O, Zahn DRT, Michaelis de Vasconcellos S, Bratschitsch R. Photoluminescence emission and Raman response of monolayer $\mathrm{MoS}_{2}, \mathrm{MoSe}_{2}$, and $\mathrm{WSe}_{2}$. Opt Express 2013, 21:49084016.

[19] Tongay S, Zhou J, Ataca C, Lo K, Matthews TS, Li J, Grossman JC, Wu J. Thermally Driven Crossover from Indirect toward Direct Bandgap in 2D Semiconductors: $\mathrm{MoSe}_{2}$ versus $\mathrm{MoS}_{2}$. Nano Lett 2012, 12:5576-5580.

[20] Gutiérrez HR, Perea-López N, Elías AL, Berkdemir A, Wang B, Lv R, López-Urías F, Crespi VH, Terrones H, Terrones M. Extraordinary Room-Temperature Photoluminescence in Triangular $\mathrm{WS}_{2}$ Monolayers. Nano Lett 2012, 13:3447-3454.

[21] Tongay S, Suh J, Ataca C, Fan W, Luce A, Kang JS, Liu J, Ko C, Raghunathanan R, Zhou J, Ogletree F, Li J, Grossman JC, Wu J. Defects activated photoluminescence in two-dimensional semiconductors: interplay between bound, charged, and free excitons. Sci Rep 2013, 3:2657.

[22] Ugeda MM, Bradley AJ, Shi S-F, da Jornada FH, Zhang Y, Qiu DY, Ruan W, Mo S.-K., Hussain Z, Shen Z-X, Wang F, Louie SG, Crommie MF. Giant bandgap renormalization and excitonic effects in a monolayer transition metal dichalcogenide semiconductor. Nat Mater
2014, 13:1091-1095.

[23] Cao T, Wang G, Han W, Ye H, Zhu C, Shi J, Niu Q, Tan P, Wang E, Liu B, Feng J. Valley-selective circular dichroism of monolayer molybdenum disulphide. Nat Commun 2012, 3:887.

[24] Zeng H, Dai J, Yao W, Xiao D, Cui X. Valley polarization in $\mathrm{MoS}_{2}$ monolayers by optical pumping. Nat Nanotechnol 2012, 7:490-493.

[25] Jones AM, Yu H, Ghimire NJ, Wu S, Aivazian G, Ross JS, Zhao B, Yan J, Mandrus DG, Xiao D, Yao W, Xu $\mathrm{X}$. Optical generation of excitonic valley coherence in monolayer $\mathrm{WSe}_{2}$. Nat Nanotech 2013, 8:634-638.

[26] Srivastava A, Sidler M, Allain AV, Lembke DS, Kis A, Imamoglu A. Valley Zeeman effect in elementary optical excitations of monolayer $\mathrm{WSe}_{2}$. Nature Phys 2015, 11:141-147.

[27] Tongay S, Fan W, Kang J, Park J, Koldemir U, Suh J, Narang D, Liu K, Ji J, Li J, Sinclair R, Wu J. Tuning Interlayer Coupling in Large-Area Heterostructures with CVD-Grown $\mathrm{MoS}_{2}$ and $\mathrm{WS}_{2}$ Monolayers. Nano Lett 2014, 14:3185-3190.

[28] Gong Y, Lin J, Wang X, Shi G, Lei S, Lin Z, Zou X, Ye G, Vajtai R, Yakobson BI, Terrones H, Terrones M, Tay BK, Lou J, Pantelides ST, Liu Z, Zhou W, Ajayan PM. Vertical and in-plane heterostructures from $\mathrm{WS}_{2} / \mathrm{MoS}_{2}$ monolayers. Nat Mater 2014, 13:1135-1142.

[29] Rivera P, Schaibley JR, Jones AM, Ross JS, Wu S, Aivazian G, Klement P, Seyler K, Clark G, Ghimire NJ, Yan J, Mandrus DG, Yao W, Xu X. Observation of long-lived interlayer excitons in monolayer $\mathrm{MoSe}_{2} \mathrm{WSe}_{2}$ heterostructures. Nat Commun 2015, 6:6242.

[30] Huo N, Kang J, Wei Z, Li S-S, Li J, Wei S-H. Novel and Enhanced Optoelectronic Performances of Multilayer $\mathrm{MoS}_{2} \mathrm{WS}_{2}$ Heterostructure Transistors. Adv Funct Mater 2014, 24:7025.

[31] Cheng R, Li D, Zhou H, Wang C, Yin A, Jiang S, Liu Y, Chen Y, Huang Y, Duan X. Electroluminescence and Photocurrent Generation from Atomically Sharp $\mathrm{WSe}_{2} / \mathrm{MoS}_{2}$ Heterojunction pn Diodes. Nano Lett 2014, 14:5590-5597.

[32] Lee C-H, Lee G-H, van Der Zande AM, Chen W, Li Y, Han M, Cui X, Arefe G, Nuckolls C, Heinz TF, Guo J, Hone J, Kim P. Atomically thin p-n junctions with van der Waals heterointerfaces. Nat Nanotechnol 2014, 9:676-681.

[33] Furchi MM, Pospischil A, Libisch F, Burgdrf̈er J, Mueller T. Photovoltaic Effect in an Electrically Tunable van der Waals Heterojunction. Nano Lett 2014, 14:4785-4791.

[34] Withers F, Del Pozo-Zamudio O, Mishchenko A, Rooney A, Gholinia A, Watanabe K, Taniguchi T, Haigh S, Geim A, Tartakovskii A, Novoselov KS. Lightemitting diodes by band-structure engineering in van der Waals heterostructures. Nat Mater 2015, 14:301306.

[35] Chernikov A, Berkelbach TC, Hill HM, Rigosi A, Li Y, Aslan OB, Reichman DR, Hybertsen MS, Heinz TF. Exciton Binding Energy and Nonhydrogenic Rydberg Series in Monolayer $\mathrm{WS}_{2}$. Phys Rev Lett 2014, 113:076802.

[36] Ye Z, Cao T, OBrien K, Zhu H, Yin X, Wang Y, Louie SG, Zhang X. Probing excitonic dark states in singlelayer tungsten disulphide. Nature 2014, 513:214.

[37] He K, Kumar N, Zhao L, Wang Z, Mak KF, Zhao H, Shan J. Tightly Bound Excitons in Monolayer $\mathrm{WSe}_{2}$. 
Phys Rev Lett 2014, 113:026803.

[38] Mak KF, He K, Lee C, Lee GH, Hone J, Heinz TF, Shan J. Tightly bound trions in monolayer $\mathrm{MoS}_{2}$. Nat Mater 2013, 12:207-211.

[39] Ross JS, Wu S, Yu H, Ghimire NJ, Jones AM, Aivazian G, Yan J, Mandrus DG, Xiao D, Yao W, Xu X. Electrical control of neutral and charged excitons in a monolayer semiconductor. Nat Commun 2013, 4:1474.

[40] Mitioglu AA, Plochocka P, Jadczak JN, Escoffier W, Rikken GLJA, Kulyuk L, Maude DK. Optical manipulation of the exciton charge state in single-layer tungsten disulfide. Phys Rev B 2013, 88:245403.

[41] Mai C, Barrette A, Yu YF, Semenov YG, Kim KW, Cao LY, Gundogdu K. Many-Body Effects in Valleytronics: Direct Measurement of Valley Lifetimes in Single-Layer $\mathrm{MoS}_{2}$. Nano Lett 2014, 14:202-206.

[42] Shang JZ, Shen XN, Cong CX, Peimyoo N, Cao BC, Eginligil M, Yu T. Observation of Excitonic Fine Structure in a 2D Transition-Metal Dichalcogenide Semiconductor. ACS Nano 2015, 9:647-655.

[43] You Y, Zhang X-X, Berkelbach TC, Hybertsen MS, Reichman DR, Heinz TF. Observation of biexcitons in monolayer $\mathrm{WSe}_{2}$. Nat Phys 2015, 11:477.

[44] Lin Y, Ling X, Yu L, Huang S, Hsu AL, Lee YH, Kong J, Dresselhaus MS, and Palacios T. Dielectric Screening of Excitons and Trions in Single-Layer $\mathrm{MoS}_{2}$. Nano Lett 2014, 14:5569-5576.

[45] Latini S, Olsen T, Thygesen KS. Excitons in van der Waals heterostructures: The important role of dielectric screening. Phys Rev B 2015, 92:245123.

[46] Ceperley D, Alder B. Ground State of the Electron Gas by a Stochastic Method. Phys Rev Lett 1980, 45:566.

[47] Perdew JP, Burke K, Ernzerhof M. Generalized Gradient Approximation Made Simple. Phys Rev Lett 1996, $77: 3865$.

[48] Perdew JP, Burke K, Ernzerhof M. Generalized Gradient Approximation Made Simple [Phys. Rev. Lett. 77, 3865 (1996)]. Phys Rev Lett 1997, 78:1396.

[49] Onida G, Reining L, Rubio A. Electronic excitations: density-functional versus many-body Green's-function approaches. Reviews of modern physics 2002, 74:601659.

[50] Heyd J, Scuseria G, Ernzerhof M. Hybrid functionals based on a screened Coulomb potential. J Chem Phys 2003, 118:8207-8215.

[51] Shishkin M, Kresse G. Implementation and performance of the frequency-dependent GW method within the PAW framework. Phys Rev B 2006, 74:035101

[52] Shishkin M, Kresse G. Self-consistent GW calculations for semiconductors and insulators. Phys Rev B 2007, $75: 235102$.

[53] Fuchs F, Furthmüller J, Bechstedt F, Shishkin M, Kresse G. Quasiparticle band structure based on a generalized Kohn-Sham scheme. Phys Rev B 2007, $76: 115109$

[54] Shishkin M, Marsman M, Kresse G. Accurate Quasiparticle Spectra from Self-Consistent GW Calculations with Vertex Corrections. Phys Rev Lett 2007, 99:246403.

[55] Gonze X, Amadon B, Anglade PM, Beuken JM, Bottin F, Boulanger P, Bruneval F, Caliste D, Caracas R, Côté M, Deutsch T, Genovese L, Ghosez P, Giantomassi M, Goedecker S, Hamann DR, Hermet P, Jollet F, Jomard G, Leroux S, Mancini M, Mazevet S, Oliveira M, Onida G, Pouillon Y, Rangel T, Rignanese GM, San- galli D, Shaltaf R, Torrent M, Verstraete M, Zerah G, Zwanziger J. ABINIT: First-principles approach to material and nanosystem properties. Comput Phys Commun 2009, 180:2582.

[56] Marini A, Hogan C, Grning M, Varsano D. yambo: An ab initio tool for excited state calculations. Comp Phys Comm, 2009 180:1392.

[57] Giannozzi P, Baroni S, Bonini N, Calandra M, Car R, Cavazzoni C, Ceresoli D, Chiarotti GL, Cococcioni M, Dabo I, Dal Corso A, de Gironcoli S, Fabris S, Fratesi G, Gebauer R, Gerstmann U, Gougoussis C, Kokalj A, Lazzeri M, Martin-Samos L, Marzari N, Mauri F, Mazzarello R, Paolini S, Pasquarello A, Paulatto L, Sbraccia C, Scandolo S, Sclauzero G, Seitsonen AP, Smogunov A, Umari P, Wentzcovitch RM. QUANTUM ESPRESSO: a modular and open-source software project for quantum simulations of materials. J Phys Condens Matter 2009, 21:395502.

[58] Kresse G, Hafner J. Ab initio molecular dynamics for liquid metals. Phys Rev B 1993, 47:558(R).

[59] Kresse G, Hafner J. Ab initio molecular-dynamics simulation of the liquid-metal?amorphous-semiconductor transition in germanium. Phys Rev B 1994, 49:14251

[60] Kresse G, Furthmüller. Efficiency of ab-initio total energy calculations for metals and semiconductors using a plane-wave basis set. J Comput Mat Sci 1996, 6:15-50.

[61] Kresse G, Furthmüller J. Efficient iterative schemes for ab initio total-energy calculations using a plane-wave basis set. Phys Rev B 1996, 54:11169.

[62] Albrecht S, Reining L, Del Sole R, Onida G. Ab Initio Calculation of Excitonic Effects in the Optical Spectra of Semiconductors. Phys Rev Lett 1998, 80:4510.

[63] Rohlfing M, Louie SG. Electron-Hole Excitations in Semiconductors and Insulators. Phys Rev Lett 1998, 81:2312.

[64] Yang L, Deslippe J, Park CH, Cohen ML, Louie SG. Excitonic Effects on the Optical Response of Graphene and Bilayer Graphene. Phys Rev Lett 2009, 103:186802.

[65] Trevisanutto PE, Holzmann M, Côté M, Olevano V. $\mathrm{Ab}$ initio high-energy excitonic effects in graphite and graphene. Phys Rev B 2010, 81:121405(R).

[66] Chen Z, Wang X-Q. Stacking-dependent optical spectra and many-electron effects in bilayer graphene. Phys Rev B 2011, 83:081405(R).

[67] Havener RW, Liang Y, Brown L, Yang L, Park J. Van Hove Singularities and Excitonic Effects in the Optical Conductivity of Twisted Bilayer Graphene. Nano Lett 2014, 14:3353-3357.

[68] Yan J-A, Ruan WY, Chou MY. Enhanced optical conductivity induced by surface states in ABC-stacked fewlayer graphene. Phys Rev B 2011, 83:245418.

[69] Yang L. Excitons in intrinsic and bilayer graphene. Phys Rev B 2011, 83:085405.

[70] Mak KF, Shan J, Heinz TF. Seeing Many-Body Effects in Single- and Few-Layer Graphene: Observation of Two-Dimensional Saddle-Point Excitons. Phys Rev Lett 2011, 106:046401.

[71] Fano U. Effects of Configuration Interaction on Intensities and Phase Shifts. Phys Rev 1961, 124:1866.

[72] Phillips JC. Ultraviolet Absorption of Insulators. III. fcc Alkali Halides. Phys Rev 1964, 136:A1705.

[73] Chae D-H, Utikal T, Weisenburger S, Giessen H, Klitzing Kv, Lippitz M, Smet J. Excitonic Fano Resonance in Free-Standing Graphene. Nano Lett 2011, 11:1379. 
[74] Wachsmuth P, Hambach R, Kinyanjui MK, Guzzo M, Benner G, Kaiser U. High-energy collective electronic excitations in free-standing single-layer graphene. Phys Rev B 2013, 88:075433.

[75] Yang L. First-principles study of the optical absorption spectra of electrically gated bilayer graphene. Phys Rev B 2010, 81:155445.

[76] Mohan B, Kumar A, Ahluwalia PK. A first principle study of interband transitions and electron energy loss in mono and bilayer graphene: Effect of external electric field. Physica E 2012, 44:1670.

[77] Rayson MJ, Briddon PR. Rapid iterative method for electronic-structure eigenproblems using localised basis functions. Comput Phys Commun 2008, 178:128-134.

[78] Pereira VM, Ribeiro RM, Peres NMR, Neto AHC. Optical properties of strained graphene. EPL 2010, 92:67001.

[79] Yang L. Excitonic Effects on Optical Absorption Spectra of Doped Graphene. Nano Lett 2011, 11:3844.

[80] Gao Y, Yuan Z. Anisotropic low-energy plasmon excitations in doped graphene: An ab initio study. Solid State Commun 2011, 151:1009.

[81] Despoja V, Novko D, Dekanić K, Sunjić M, Marušić L. Two-dimensional and $\pi$ plasmon spectra in pristine and doped graphene. Phys Rev B 2013, 87:075447.

[82] Kadi F, Malic E. Optical properties of Bernal-stacked bilayer graphene: A theoretical study. Phys Rev B 2014, 89:045419.

[83] Singh N, Kaloni TP, Schwingenschlögl U. A firstprinciples investigation of the optical spectra of oxidized graphene. Appl Phys Lett 2013, 102:023101.

[84] Sedelnikova OV, Bulusheva LG, Okotrub AV. Ab initio study of dielectric response of rippled graphene. J Chem Phys 2011, 134:244707.

[85] Yang L, Cohen ML, Louie SG. Excitonic Effects in the Optical Spectra of Graphene Nanoribbons. Nano Lett 2007, 7:3112-3115.

[86] Prezzi D, Varsano D, Ruini A, Marini A, Molinari E. Optical properties of graphene nanoribbons: The role of many-body effects. Phys Rev B 2008, 77:041404(R).

[87] Wang S, Wang J. Quasiparticle Energies and Optical Excitations in Chevron-Type Graphene Nanoribbon. J. Phys. Chem. C 2012 116: 10193.

[88] Yamamoto T, Noguchi T, Watanabe K. Edge-state signature in optical absorption of nanographenes: Tightbinding method and time-dependent density functional theory calculations. Phys. Rev. B 2006 74: 121409(R).

[89] Zhou A, Sheng W. Van Hove singularities in graphene nanoflakes. J. Appl. Phys. 2012 112: 094313.

[90] Luo G, Qian X, Liu H, Qin R, Zhou J, Li L, Gao Z, Wang E, Mei WN, Lu J, Li Y, Nagase S. Quasiparticle energies and excitonic effects of the two-dimensional carbon allotrope graphdiyne: Theory and experiment. Phys. Rev. B 2011 84: 075439.

[91] Huang S, Liang, Y, Yang L. Exciton spectra in twodimensional graphene derivatives. Phys. Rev. B 2013 88: 075441.

[92] Bechstedt F, Matthes L, Gori P, Pulci, O. Infrared absorbance of silicene and germanene. Appl. Phys. Lett. 2012 100: 261906.

[93] Matthes, L, Gori, P, Pulci, O, Bechstedt, F. Universal infrared absorbance of two-dimensional honeycomb group-IV crystals. Phys. Rev. B 2013 87: 035438.

[94] Cakir, D, Sahin, H, Peeters, FM. Tuning of the electronic and optical properties of single-layer black phos- phorus by strain. Phys. Rev. B 2014 90: 205421.

[95] Cakir, D, Sevik, C, Peeters, FM. Significant effect of stacking on the electronic and optical properties of fewlayer black phosphorus. Phys. Rev. B 2015 92: 165406.

[96] Seixas, L, Rodin, AS, Carvalho, A, Castro Neto, AH. Exciton binding energies and luminescence of phosphorene under pressure. Phys. Rev. B 2015 91: 115437.

[97] Tran, V, Yang, L. Scaling laws for the band gap and optical response of phosphorene nanoribbons. Phys. Rev. B 2014 89: 245407.

[98] Pulci O, Gori P, Marsili M, Garbuio V, Seitsonen AP, Bechstedt F, Cricenti A, Del Sole R. Electronic and optical properties of group IV two-dimensional materials. Phys. Status Solidi A 2010 207: 291.

[99] Cudazzo P, Attaccalite C, Tokatly V, Rubio A. Strong Charge-Transfer Excitonic Effects and the BoseEinstein Exciton Condensate in Graphane. Phys. Rev. Lett. 2010 104: 226804.

[100] Wei, W, Jacob, T. Strong charge-transfer excitonic effects in C4H-type hydrogenated graphene. Phys. Rev. B 2012 86: 165444.

[101] Cheng, JL , Salazar, C., Sipe, JE. Optical properties of functionalized graphene. Phys. Rev. B 2013 88: 045438.

[102] Samarakoon, DK, Chen, Z, Nicolas, C, Wang, XQ. Structural and Electronic Properties of Fluorographene. Small 2011 7: 965.

[103] Karlický, F, Otyepka, M. Band Gaps and Optical Spectra of Chlorographene, Fluorographene and Graphane from G0W0, GW0 and GW Calculations on Top of PBE and HSE06 Orbitals. J. Chem. Theory Comput. 2013 9: 4155.

[104] Karlický, F, Otyepka, M. Band gaps and optical spectra from single- and double-layer fluorographene to graphite fluoride: many-body effects and excitonic states. Ann. Phys. 2014 526: 408.

[105] Wei, W, Jacob, T. Electronic and optical properties of fluorinated graphene: A many-body perturbation theory study. Phys. Rev. B 2013 87: 115431.

[106] Gunasinghe, RN , Samarakoon, DK , Arampath, AB, Shashikala, HBM, Vilus, J, Hall, JH, Wang, XQ. Resonant orbitals in fluorinated epitaxial graphene. Phys. Chem. Chem. Phys. 2014 16: 18902-18906.

[107] Gourmelon, E, Lignier, O, Hadouda, H, Couturier, G, Bernede, JC, Tedd, J, Pouzed, J, Salardenne, J. MS2 $(\mathrm{M}=\mathrm{W}, \mathrm{Mo})$ photosensitive thin films for solar cells. J. Sol. Energy Mater. Sol. Cells 199746115.

[108] Mak, K, Lee, C, Hone, J, Shan, J, Heinz, TF. Atomically Thin MoS2: A New Direct-Gap Semiconductor. Phys. Rev. Lett. 2010 105: 136805.

[109] Shih, BC, Xue, Y, Zhang, P, Cohen, ML, Louie, SG. Quasiparticle Band Gap of $\mathrm{ZnO}$ : High Accuracy from the Conventional G0W0 Approach. Phys. Rev. Lett. 2010 105: 146401.

[110] Cheiwchanchamnangij T, Lambrecht WRL. Quasiparticle band structure calculation of monolayer, bilayer, and bulk MoS2. Phys. Rev. B. 2012 85: 205302.

[111] Ramasubramaniam A. Large excitonic effects in monolayers of molybdenum and tungsten dichalcogenides. Phys. Rev. B. 2012 866, 115409.

[112] Shi H, Pan H, Zhang YW, Yakobson BI. Quasiparticle band structures and optical properties of strained monolayer MoS2 and WS2. Phys. Rev. B. 2013 87: 155304.

[113] Sanchez AM, Sangalli D, Hummer K, Marini A, Wirtz L. Effect of spin-orbit interaction on the optical spectra 
of single-layer, double-layer, and bulk MoS2. Phys. Rev. B. 2013 88: 045412.

[114] Qiu DY, Jornada FH, Louie SG. Optical Spectrum of MoS2: Many-Body Effects and Diversity of Exciton States. Phys. Rev. Lett. 2013 111: 216805.

[115] Feng J, Qian X, Huang CW, Li J. Strain-engineered artificial atom as a broad-spectrum solar energy funnel. Nature Photonics 2012 6: 866.

[116] Joswig JO, Lorenz T, Wendumu TB, Gemming, S, Seifert, G. Optics, Mechanics, and Energetics of TwoDimensional MoS2 Nanostructures from a Theoretical Perspective. Accounts of Chemical Research 2015 48: 48-55.

[117] Jing Y, Tan X, Zhou Z, Shen P. Tuning electronic and optical properties of MoS2 monolayer via molecular charge transfer. J. Mater. Chem. A 2014 2: 16892.

[118] Wie JW, Ma ZW, Zeng H, Wang ZY, Wei Q, Peng P. Electronic and optical properties of vacancy-doped WS2 monolayers. AIP Advances 2012 2: 042141.

[119] Yuan S, Roldán R, Katsnelson MI, Guinea F. Effect of point defects on the optical and transport properties of MoS2 and WS2. Phys. Rev. B 2014 90: 041402(R).

[120] Feng LP, Su J, Liu ZT. Effect of vacancies on structural, electronic and optical properties of monolayer MoS2: A first-principles study. Journal of Alloys and Compounds 2014 613: 122.

[121] He J, Hummer K, Franchini C. Stacking effects on the electronic and optical properties of bilayer transition metal dichalcogenides MoS2, MoSe2, WS2, and WSe2. Phys. Rev. B 2014 89: 075409.

[122] Yan J, Jacobsen KW, Thygesen KS. Optical properties of bulk semiconductors and graphene/boron nitride: The Bethe-Salpeter equation with deriva- tive discontinuity-corrected density functional energies. Phys. Rev. B 2012 86: 045208.

[123] Attaccalite C, Bockstedte M, Marini A, Rubio A, Wirtz L. Coupling of excitons and defect states in boronnitride nanostructures. Phys. Rev. B 2011 83, 144115.

[124] Wang J, Xu Y, Chen H, Zhang B. Ultraviolet dielectric hyperlens with layered graphene and boron nitride. J. Mater. Chem. 2012 22: 15863-15868.

[125] Bernardi M, Palummo M, Grossman JC. Optoelectronic Properties in Monolayers of Hybridized Graphene and Hexagonal Boron Nitride. Phys. Rev. Lett. 2012 108: 226805.

[126] Komsa HP, Krasheninnikov AV. Electronic structures and optical properties of realistic transition metal dichalcogenide heterostructures from first principles. Phys. Rev. B 2013 88: 085318.

[127] Kang J, Tongay S, Zhou J, Li J, Wu J. Band offsets and heterostructures of two-dimensional semiconductors. J. Appl. Phys. Lett. 2013 102: 012111.

[128] Debbichi L, Eriksson, O Lebegue S. Electronic structure of two-dimensional transition metal dichalcogenide bilayers from ab initio theory. Phys. Rev. B 2014 89: 205311.

[129] Palummo M, Bernardi M, Grossman JC. Exciton Radiative Lifetimes in Two-Dimensional Transition Metal Dichalcogenides. Nano Lett. 2015 15: 2794-2800

[130] Fang H, Battaglia C, Carraro C, Nemsak S, Ozdol B, Kang JS, Bechtel HA, Desai SB, Kronast F, Unal AA, Conti G, Conlon C, Palsson GK, Martin MC, Minor AM, Fadley CS, Yablonovitch E, Maboudian R, Javey A. Strong interlayer coupling in van der Waals heterostructures built from single-layer chalcogenides. PNAS 2014 111: 6198-6202 